Citation: Hirt C, Featherstone WE, Claessens SJ (2011) On the accurate numerical evaluation of geodetic convolution integrals, Journal of Geodesy, online first. doi: 10.1007/s00190-011-0451-5. 


\title{
On the accurate numerical evaluation of geodetic convolution integrals
}

\section{Hirt}

Western Australian Centre for Geodesy \& The Institute for Geoscience Research Curtin University of Technology, GPO Box U1987, Perth, WA 6845, Australia Fax: +61 89266 2703; Email: c.hirt@curtin.edu.au

\section{W.E. Featherstone}

Western Australian Centre for Geodesy \& The Institute for Geoscience Research Curtin University of Technology, GPO Box U1987, Perth, WA 6845, Australia Fax: +61 89266 2703; Email: w.featherstone@curtin.edu.au

\section{S.J. Claessens}

Western Australian Centre for Geodesy \& The Institute for Geoscience Research Curtin University of Technology, GPO Box U1987, Perth, WA 6845, Australia Fax: +61 89266 2703; Email: s.claessens@curtin.edu.au

\begin{abstract}
In the numerical evaluation of geodetic convolution integrals, whether by quadrature or discrete/fast Fourier transform (D/FFT) techniques, the integration kernel is sometimes computed at the centre of the discretised grid cells. For singular kernels - a common case in physical geodesy - this approximation produces significant errors near the computation point, where the kernel changes rapidly across the cell. Rigorously, mean kernels across each whole cell are required. We present one numerical and one analytical method capable of providing estimates of mean kernels for convolution integrals. The numerical method is based on Gauss-Legendre quadrature (GLQ) as efficient integration technique. The analytical approach is based on kernel weighting factors, computed in planar approximation close to the computation point, and used to convert non-planar kernels from point to mean representation. A numerical study exemplifies the benefits of using mean kernels in Stokes's integral. The method is validated using closed-loop tests based on the EGM2008 global gravity model, revealing that using mean kernels instead of point kernels reduces numerical integration errors by a factor of $\sim 5$ (at a grid-resolution of 10 arc minutes). Analytical mean kernel solutions are then derived for 14 other commonly used geodetic convolution integrals: Hotine, Eötvös, Green-Molodensky, tidal displacement, ocean tide loading, deflection-geoid, Vening-Meinesz, inverse Vening-Meinesz, inverse Stokes, inverse Hotine, terrain correction, primary indirect effect, Molodensky's G1 term and the Poisson integral. We recommend that mean kernels be used to accurately evaluate geodetic convolution integrals, and the two methods presented here are effective and easy to implement.
\end{abstract}

Keywords: Convolution integrals, mean kernel, point kernel, numerical integration, GaussLegendre quadrature, kernel weighting factor 


\section{Introduction}

Convolution integrals play an important role in geodesy, amongst many other disciplines. For instance, they establish the connection between numerous functionals of the Earth's external gravitational field (e.g., Schwarz et al. 1990), but are also used in ocean tide loading computations (e.g., Bos and Baker 2005). Convolution integrals can be computed by quadrature techniques (e.g., Lehmann 1997; Huang et al. 2000) in the space domain or by Discrete/Fast Fourier Transform (D/FFT) techniques in the frequency domain (e.g., Haagmans et al. 1993; Schwarz et al. 1990), but this is only a computational preference since both techniques are inevitably subject to approximation. This is because numerical integration requires discretisation of the observations or other data (herein called the functional) over small-as-possible surface elements (herein called cells) (cf. Heiskanen and Moritz 1967, p117; Torge 2001, p284). For each cell, it is necessary to utilise representative estimates of both the functional and the integration kernel. In general, this requires not only the functional, but also the kernel to be averaged over the surface area of each cell (Heiskanen and Moritz 1967, p119). In other words, mean values of both the functional and the kernel across each cell are required.

Sometimes, the point value of the kernel is evaluated at the centre of the cell, but - as we will show here - this may be a coarse approximation, especially for the singular convolution integrals commonly encountered in geodesy and other areas. Particularly in the close vicinity of the computation point, the kernels in convolution integrals may change rapidly across the cell and can therefore not be approximated through centre-of-cell values with sufficient accuracy (Vaníček and Krakiwsky 1986, p543, Strang van Hees 1990, p238). Beyond a certain distance from the computation point, most geodetic kernels are reasonably constant over a cell so that centre-of-cell kernel values are a good enough approximation. However, if the kernel behaves in a rapidly varying way in other areas of the integration domain, then the techniques presented here should also be considered.

Centre-of-cell kernel values may be sufficiently accurate if a suitably dense grid of the functional is used. Densification of the functional field can be achieved through interpolation prior to the numerical integration. However, this approach is not treated in the present paper because the additional computational requirements due to the grid densification may be significant compared to the mean kernel computation strategies presented here.

As further justification, a recent textbook on geodesy does not fully acknowledge the necessity for the computation of mean kernel values by saying "For this purpose [of numerical integration], either a set of gridded point anomalies is formed from the observed [gravity field] data [...] or mean values over surface blocks delineated by meridians and parallels are calculated. The latter case also requires the integration of the Stokes or Vening-Meinesz function over the block." (Torge 2001, p284). However, also the first case requires the kernel to be integrated over the block, so as to avoid an unnecessary approximation (cf. Heiskanen and Moritz 1967, p119, Vaníček and Krakiwsky 1986, p543). 
In many studies concerned with the numerical evaluation of geodetic convolution integrals, the necessity for whole-of-cell mean values of the kernel (herein abbreviated to mean kernels) is rarely acknowledged, or at least the details of the computational algorithms are omitted or glossed over. Exceptions are, e.g., Strang van Hees 1990; de Min 1994, who developed analytical approaches to compute mean kernels of Stokes's function (also see Kearsley 1986; de Min 1996; Featherstone and Olliver 1997; Boyarksy et al. 2010). Numerical approaches for accurate evaluation of geodetic integrals were described, e.g., by Alberts and Klees (2004) and van Gysen (1994). Alberts and Klees (2004) used Gaussian quadrature for evaluation of airborne gravity integrals and van Gysen (1994) describes a spline quadrature approach for evaluation of Stokes, Vening-Meinesz and terrain-related integrals. Other examples of works with focus on numerical integration methods for geodetic integrals are Klees (1996) and Lehmann (1997).

Here we present and compare two general approaches for the improved numerical evaluation of convolution integrals by using mean kernels. This can be applied to both quadrature and D/FFT techniques. Initially, the paper sets a framework for the evaluation of geodetic convolution integrals and describes the numerical and analytical mean kernel integration in a generalised manner (Sect. 2). As the numerical integration method, we use the efficient Gauss-Legendre quadrature (also see Appendix A1). The analytical integration method is based on the concept of kernel weighting factors, which are derived based on planar kernel approximations. In Sect. 3, a case study is presented for the accurate evaluation of Stokes's integral. This investigates the computation of mean kernel values for Stokes's integral through analytical and numerical integration methods, and the results of both methods are compared to each other and to point kernels. It relies on closed-loop tests with assumed error-free data to demonstrate the importance of mean kernels. Section 4 then presents the analytical solutions for the mean kernel computation for 14 additional geodetic convolution integrals. If an integral has been omitted from this list, then mean kernel values can be obtained using the principles presented herein.

\section{A generalised analytical approach for mean kernel computation}

\subsection{Basic form of convolution integrals}

Many convolution integrals establish a relation between a functional $f(\varphi, \lambda)$ that is available at points $Q$ over a certain integration domain, and a target quantity $e\left(\varphi_{P}, \lambda_{P}\right)$ at a computation point $P$. In generalised form and spherical approximation (as is often still used in many areas of geodesy), the convolution integral is (after van Gelderen 1991)

$$
e\left(\varphi_{P}, \lambda_{P}\right)=k \iint_{\sigma} f(\varphi, \lambda) \cdot Y(\psi) d \sigma
$$

where $k$ is some constant, $\sigma$ is the integration domain (often the surface of a whole sphere) and $d \sigma$ are infinitesimally small surface compartments. The quantity $Y(\psi)$ denotes the integration kernel, which is a function of the spherical distance $\psi$ between computation point $P$ and integration point $Q$. The kernel $Y(\psi)$ is mostly computed with closed-form 
expressions, but series expansions can also be used provided they are of sufficient accuracy. The spherical distance $\psi$ can be computed via (Strang van Hees 1990, p236):

$\sin ^{2}\left(\frac{\psi}{2}\right)=\sin ^{2}\left(\frac{\varphi_{P}-\varphi_{Q}}{2}\right)+\sin ^{2}\left(\frac{\lambda_{P}-\lambda_{Q}}{2}\right) \cos \varphi_{P} \cos \varphi_{Q}$

with $\left(\varphi_{P}, \lambda_{P}\right)$ being the spherical coordinates of the computation point $P$ and $\left(\varphi_{Q}, \lambda_{Q}\right)$ the spherical coordinates of the data point $Q$. Alternatively to Eq. (2), the spherical cosine rule can be used, but which is subject to larger rounding errors for very small $\psi$.

Practically, convolution integrals are evaluated numerically by means of summation of the products $f\left(\varphi_{Q}, \lambda_{Q}\right) \cdot Y(\psi)$ over small-as-practically-possible yet finite cells, the size of which is often dictated by the spatial resolution of the functional under consideration. The discretisation of the Earth's surface is usually achieved by means of graticules along meridians and parallels, giving a subdivision into a total of $n$ small, approximately rectangular cells $q_{i}$ (Heiskanen and Moritz 1967, p118):

$e\left(\varphi_{P}, \lambda_{P}\right)=k \sum_{i=1}^{n} \iint_{q_{i}} f(\varphi, \lambda) Y(\psi) d \sigma$

However, other approaches have been used, such as concentric rings (Hammer 1939; Heiskanen and Moritz 1967, p118; Kearsley 1986) or map-projected grid lines (e.g., Hipkin 1988). Discretised numerical evaluation of Eq. (3) demands that both $f(\varphi, \lambda)$ and $Y(\psi)$ be integrated over each cell $q_{i}$ so as to obtain the most representative values. Frequently, the whole continuous functional $f(\varphi, \lambda)$ is not available and mean values $\bar{f}_{i}$ are computed as (weighted) average of the functionals available for the cell $q_{i}$. Therefore, the fully discretised convolution integral degenerates to (after Heiskanen and Moritz 1967, p118):

$$
\begin{aligned}
e\left(\varphi_{P}, \lambda_{P}\right) & \approx k \sum_{i=1}^{n} \bar{f}_{i} \iint_{q_{i}} Y(\psi) d \sigma \\
& =k \sum_{i=1}^{n} \bar{f}_{i} d_{i}
\end{aligned}
$$

with

$d_{i}=\iint_{q_{i}} Y(\psi) d \sigma \approx \bar{Y}_{i} \cos \varphi_{Q}, \Delta \varphi \Delta \lambda$.

where $\bar{Y}_{i}$ is the mean value of the integral kernel across the area approximated with $\cos \varphi_{Q} \Delta \varphi \Delta \lambda$ of cell $q_{i}$ and $\varphi_{Q^{\prime}}$ is the latitude of the centre point $Q^{\prime}$ of the cell. In the sequel, the index $i$ will be dropped for kernels as it is clear that these refer to single cells. The computation methods for the mean values $\bar{f}_{i}$ of the gravity field functionals are not within 
the scope of the present study. See, e.g., Tscherning (2003) for theoretical aspects of mean functional values and, e.g., Featherstone et al. (2010) and Claessens et al. (2011) for recent practical computations of mean gravity anomalies.

\subsection{The necessity for mean kernels}

From Eq. (5), it follows that mean kernel estimates $\bar{Y}$ need to be utilised for each cell. Sometimes, the kernel $Y(\psi)$, evaluated at the centre of the cell $q_{i}$, is introduced as a firstorder estimate for the mean kernel

$\bar{Y} \approx Y(\psi)$,

but this is an approximation, which can be too crude at times. According to Heiskanen and Moritz (1967, p119), such a centre-of-cell approximation is admissible under the condition that the integral kernel is "reasonably constant over the compartment". However, in the inner zone (this is the neighbourhood of the computation point), where many singular kernels vary rapidly across the cell, this centre-of-cell approximation is not sufficiently accurate. For these cells, the mean kernel $\bar{Y}$ must be calculated as an integral mean over the whole cell (e.g., Vaníček and Krakiwsky 1986, p543).

For a cell $q_{i}$ of size of $\Delta \lambda$ by $\Delta \varphi$, bounded by meridians $\lambda_{1}, \lambda_{2}$ and parallels $\varphi_{1}, \varphi_{2}$ $\left(\lambda_{1}<\lambda_{2}\right.$ and $\varphi_{1}<\varphi_{2}$ ), the mean kernel value is formally computed from (after Vaníček and Krakiwsky 1986, p543; de Min 1994):

$\bar{Y}(u)=\frac{1}{\Delta \sigma} \int_{\lambda=\lambda_{1}}^{\lambda_{2}} \int_{\varphi=\varphi_{1}}^{\varphi_{2}} Y(\psi) \cos \varphi d \varphi d \lambda$

where $u=\left(\lambda_{1}, \varphi_{1}, \lambda_{2}, \varphi_{2}\right)$ is a vector containing the corner coordinates of the cell $q_{i}, \psi$ is the spherical distance between the computation point $\left(\varphi_{P}, \lambda_{P}\right)$ and the integration point $(\varphi, \lambda)$ under evaluation and $\Delta \sigma=\cos \varphi_{Q}, \Delta \varphi \Delta \lambda$ is the (approximated) surface area of the cell. The cell boundaries $u$ are computed from:

$\lambda_{1}=\lambda_{Q^{\prime}}-\Delta \lambda / 2, \quad \varphi_{1}=\varphi_{Q^{\prime}}-\Delta \varphi / 2$,

$\lambda_{2}=\lambda_{Q^{\prime}}+\Delta \lambda / 2, \quad \varphi_{2}=\varphi_{Q^{\prime}}+\Delta \varphi / 2$.

Basically, there are two different strategies capable of providing mean kernel estimates:

- $\quad$ Numerical integration of the kernel $Y(\psi)$ over a cell $q_{i}$ is relatively straightforward. The kernel $Y(\psi)$ is computed at a number of evaluation points across the cell $q_{i}$. The arithmetic or weighted average of these evaluations represents a numerical estimate for the mean kernel $\bar{Y}(u)$ in cell $q_{i}$. One of the most efficient numerical integration techniques is the Gauss-Legendre quadrature technique, which uses a weighted average of appropriately selected evaluation points (see Sect. 2.3 and appendix A1). 
- Analytical integration: Because closed-form expressions of geodetic kernels are often nested combinations of trigonometric and logarithmic functions, the main difficulty in analytical integration arises from finding the antiderivative (cf. de Min 1994), if possible at all. However, planar approximations of the geodetic kernels can be analytically integrated in most cases. They are utilised here to derive so-called kernel weighting factors, which allow conversion from point values of the non-planar geodetic kernel to mean values (Sect. 2.4)

\subsection{Numerical mean kernel computation through Gauss-Legendre quadrature}

For the numerical computation of cell-mean kernels, a range of numerical integration methods can be used, such as the rectangular method, Simpson's method, Monte Carlo integration, Gauss-Legendre Quadrature and others (e.g., Stark 1970; Abramowitz and Stegun 1972; Conte and de Boor 1972, Stoer and Bulirsch 1980, Hamming 1986). The mean-value theorem for integration is routinely used to estimate an upper bound of the error committed in the numerical integration.

Gauss-Legendre Quadrature (GLQ) is used here because it belongs to the most efficient numerical integration techniques (e.g., Stark 1970, Sormann 2009), in that, only a small number of evaluation nodes is needed for a rapid convergence against the 'true' mean value. In the geodetic context, GLQ has been applied, e.g., by Alberts and Klees (2004) and Lehmann (1997) for the evaluation of surface integrals; by Asgharzadeh et al. (2007), WildPfeiffer (2008) and Sampietro et al. (2007) for forward modelling of gravity effects generated by geometric bodies, and by Makhloof and Ilk (2008) for the computation of truncation coefficients in gravimetric terrain effect computations.

GLQ uses specially selected evaluation points at which the geodetic integral kernel is computed and weighted to give highly-accurate mean kernel estimates. The evaluation points are the zero-crossings of the Legendre polynomials (e.g. Hamming 1986, p455), transformed to the integration domain $\left[\lambda_{1} \lambda_{2}\right] \times\left[\varphi_{1} \varphi_{2}\right]$. GLQ numerical mean kernels are computed through (see Appendix A1 for a derivation):

$$
\bar{Y}_{G L Q}=\frac{1}{\sum w_{i} w_{j}} \sum_{i=1}^{n} \sum_{j=1}^{m} w_{i} w_{j} Y\left(\psi\left(\varphi_{P}, \lambda_{P}, \varphi_{Q^{\prime} j}, \lambda_{Q^{\prime} i}\right)\right)
$$

where $n=m$ is the quadrature degree, $w_{i}, w_{j}$ are the so-called Gaussian weights and $\left(\varphi_{Q^{\prime} i}, \lambda_{Q^{\prime} j}\right.$ ) are the evaluation points, the locations of which correspond to the zero-crossings of the Legendre polynomials (see Appendix A1 and Fig. 1). The computation of the Gaussian weights $w_{i}, w_{j}$ and of the coordinates $\left(\varphi_{Q^{\prime} i}, \lambda_{Q^{\prime} j}\right)$ is detailed in Appendix A1. For a GLQ quadrature degree $n$, a total of $n^{2}$ point evaluations of the geodetic kernel $Y(\psi)$ are carried out. The convergence behaviour of GLQ mean kernels is further investigated in our case study on Stokes's integral (Sect. 3).

\subsection{Analytical mean kernel computation through kernel weighting factors}


A pragmatic approach to the analytical computation of cell-mean values of any arbitrary kernel $Y(\psi)$ uses the planar approximation of the convolution integral, in the vicinity of the computation point for rapidly varying kernels. For many geodetic convolution integrals, planar forms have already been published (e.g., Schwarz et al. 1990; Sideris and Li 1993; El Habiby 2007) and these will be utilised here to aid in finding analytical expressions for mean kernels; if not, new derivations are presented (Sect. 4). Importantly, we do not use the planar approximations as a substitute for the spherical kernels; the only purpose of the planar forms is to facilitate the conversion from point to cell-mean kernels.

The approach presented next is an extension of the works of Strang van Hees (1990) and de Min (1994); also see Featherstone and Olliver (1997). To yield mean kernel estimates, Strang van Hees (1990) and de Min (1994) analytically integrated the planar approximation of Stokes's function. Strang van Hees (1990) used a 1D-analytical integration (1D-AI) approach, assuming square-shaped cells, while de Min (1994) presented a 2Danalytical integration (2D-AI) approach that is more generalised. Here, we adopt the 2D-AI that properly accounts for the possibility of rectangularly shaped cells in areas of high latitude, giving more accurate results (Sect. 3.5). The 2D-AI is combined with the kernel weighting factors (ratio between mean and point kernels) introduced by Strang van Hees (1990).

For the planarisation, we introduce right-rectangular coordinates $(x, y)$ that describe distances between the computation point $P$ and the data points $Q$ in planar approximation, while also accounting for meridional convergence:

$$
\begin{aligned}
& x=\left(\lambda_{Q}-\lambda_{P}\right) \cos \varphi_{Q}, \\
& y=\varphi_{Q}-\varphi_{P} .
\end{aligned}
$$

The origin of the right-rectangular coordinate system is the computation point $P$. Let us further introduce the kernel $K(x, y)$ as the planar approximation of the (usually spherical) kernel $Y(\psi)$ in the integration domains where it must be adapted. In planar approximation, mean kernel values $\bar{K}(v)$ are obtained as integral means across the cell (after Vaníček and Krakiwsky 1986, p543; de Min 1994):

$$
\bar{K}(v)=\frac{1}{b} \int_{x=x_{1}}^{x_{2}} \int_{y=y_{1}}^{y_{2}} K(x, y) d x d y
$$

where vector $v=\left(x_{1}, y_{1}, x_{2}, y_{2}\right)$ contains the boundaries of cell $q_{i}$ relative to the computation point $P$ :

$$
\begin{aligned}
& y_{1}=\varphi_{Q^{\prime}}-\varphi_{P}-\frac{\Delta \varphi}{2}, x_{1}=\left(\lambda_{Q^{\prime}}-\lambda_{P}-\frac{\Delta \lambda}{2}\right) \cos \varphi_{Q^{\prime}}, \\
& y_{2}=\varphi_{Q^{\prime}}-\varphi_{P}+\frac{\Delta \varphi}{2}, x_{2}=\left(\lambda_{Q^{\prime}}-\lambda_{P}+\frac{\Delta \lambda}{2}\right) \cos \varphi_{Q^{\prime}}
\end{aligned}
$$


The surface area of cell $q_{i}$ in planar approximation is $a=\left(x_{2}-x_{1}\right)\left(y_{2}-y_{1}\right)$. As opposed to the often-spherical integral kernel $Y(\psi)$, antiderivatives $F(x, y)$ can be found (shown later) for the planar kernel $K(x, y)$, allowing us to solve Eq. (11) as:

$\bar{K}(v)=\frac{1}{a} \|\left.\left. F(x, y)\right|_{y_{1}} ^{y_{2}}\right|_{x_{1}} ^{x_{2}}$

Importantly, our strategy is not to use planar mean kernel values $\bar{K}(v)$ as a complete substitute for mean kernels $\bar{Y}(u)$ over the entire integration domain. This is because the planarisation deteriorates the accuracy of the numerical integration at some distance from the computation point. Instead, we apply the following 'trick' that accurately gives mean kernels $\bar{Y}(u)$, while simultaneously preserving the characteristics of the original kernel $Y(\psi)$. Applying a trivial multiplication, the mean kernel $\bar{Y}(u)$ is

$\bar{Y}(u)=\frac{\bar{Y}(u)}{Y(\psi)} Y(\psi) \quad, Y(\psi) \neq 0$

where $\bar{Y}(u) / Y(\psi)$ is a factor expressing the ratio between the whole-of-cell-mean and centreof-cell point value kernel for cell $q_{i}$. This factor is termed the kernel weighing factor, also known as the integration weighting factor (cf. Featherstone and Olliver 1997, Strang van Hees 1990).

The kernel weighting factor can analogously be computed in planar approximation, which is $\bar{K}(v) / K(x, y)$. In cases when the planar kernel $K(x, y)$ is a reasonably good approximation of $Y(\psi)$ near the computation point $P$, the kernel weighting factors $\bar{Y}(u) / Y(\psi)$ and $\bar{K}(v) / K(x, y)$ for a given cell are almost identical:

$\frac{\bar{Y}(u)}{Y(\psi)} \approx \frac{\bar{K}(v)}{K(x, y)}$.

Confirmation of the validity of Eq. (15) will be obtained from the numerical tests in Sect. 3.

Rearranging Eq. (15) allows convenient conversion from point kernels $Y(\psi)$ to mean kernels $\bar{Y}(u)$

$\bar{Y}(u) \approx \frac{\bar{K}(v)}{K(x, y)} Y(\psi)$.

Consequently, kernel weighting factors $\bar{K}(v) / K(x, y)$ represent an efficient aid to scale from point kernels $Y(\psi)$ to mean kernels $\bar{Y}(u)$, without the necessity to analytically integrate $Y(\psi)$ (see the numerical study in Sect. 3). It should be emphasised that the planar 
approximations are merely used as auxiliary means in the computation of kernel weighting factor and not as substitute for the original kernel.

\section{Case-study example: Stokes's integral}

From a range of convolution integrals (cf. Sect. 4), we have selected Stokes's integral for our case study, not only for reasons of convenience, but also because it is a commonly used geodetic convolution integral. However, exactly the same principles can be applied to the other integrals in Sect. 4, or any other convolution integral for that matter. The aims of this case study are to: (i) exemplify and validate the numerical and analytical computation of mean kernels, (ii) investigate the characteristics of mean kernels, and (iii) present closed-loop test results demonstrating that the computation of mean kernels does play a key role in the accurate numerical evaluation of convolution integrals.

\subsection{Stokes' integral}

Stokes's integral establishes the relation between gravity anomalies $\Delta g$ over the globe and the disturbing potential $T$ at a point, which is converted to the geoid undulation $N$ by Bruns's formula. Convolution integration of continuously given gravity anomalies $\Delta g$, available for (infinitesimally) small compartments $d \sigma$, over the whole surface of the Earth $\sigma$ gives in spherical approximation (Heiskanen and Moritz 1967, p94):

$$
N=\frac{R}{4 \pi \gamma} \iint_{\sigma} \Delta g \cdot S(\psi) d \sigma
$$

where $R$ is the mean Earth radius and $\gamma$ is normal gravity. Stokes's kernel $S(\psi)$, or any modification thereof, takes the role of a weighting function for the gravity anomalies depending on their spherical distance $\psi$ from the computation point. In closed form, Stokes's kernel depends on $\psi$ by (Heiskanen and Moritz 1967, p94):

$$
S(\psi)=\frac{1}{\sin (\psi / 2)}-6 \sin (\psi / 2)+1-5 \cos \psi-3 \cos \psi \ln \left[\sin (\psi / 2)+\sin ^{2}(\psi / 2)\right]
$$

Stokes's kernel is defined for $0<\psi \leq \pi$ but is singular for $\psi=0$. Notwithstanding, it is possible to compute the weight for the cell centred to the computation point (also called innermost zone) by alternative methods (Heiskanen and Moritz 1967, p122; Haagmans et al. 1993, p240).

Importantly, for small $\psi$ of up to a few arc-degrees, Stokes's kernel is dominated by its first term, which can be approximated as:

$$
S^{1 s t}(\psi)=\frac{1}{\sin (\psi / 2)} \approx \frac{2}{\psi} .
$$

This approximate relation is used below as an aid in the analytical computation of mean kernels (also see Sect. 4). 


\subsection{Analytical computation of mean Stokes kernels}

\subsubsection{One-dimensional integration}

Based on 1D-AI across the cell, Strang van Hees (1990) published a simple analytical solution to compute estimates of mean kernels. Using the crude approximation of Stokes's kernel Eq. (19) in the inner zone, Strang van Hees (1990, p238) derived a simplified kernel weighting factor

$$
W_{S v H}=\frac{\psi}{\delta} \ln \left(\frac{2 \psi+\delta}{2 \psi-\delta}\right)
$$

to convert point Stokes kernels $S(\psi)$ to mean Stokes kernels $\bar{S}_{S v H}$ by

$$
\bar{S}_{S v H}=W_{S v H} S(\psi),
$$

where $\delta$ denotes the size of a square-shaped cell and $\psi$ is the spherical distance between the innermost zone and the centre of the cell under evaluation. For example, for a 1 arc-min cell adjacent to the innermost zone in the North-South-direction $(\psi=\delta=1)$, the Strang van Hees factor $W_{S v H}$ is 1.0986. The Strang van Hees factor $W_{S v H}$ was used for the computation of mean kernel estimates by Featherstone and Olliver (1997) in a study on validation of Stokesbased geoid computation software.

An underlying assumption in Strang van Hees's (1990) approach is regular cells of equal side length $\delta$. Since mean gravity anomaly data are usually provided in terms of latitude-longitude grids, this condition only holds at and near the equator. Due to meridional convergence, such grid cells become a spherical rectangle at some distance from the equator. To work with grids at arbitrary latitudes, $\delta$ must be approximated in some way, e.g., through $\Delta \varphi$ or $\Delta \lambda \cos \varphi$. Using $\delta=\Delta \varphi$ in Eq. (20) is problematic insofar as cells adjacent to the innermost zone in the East and West directions, the denominator $2 \psi-\Delta \varphi$ becomes 0 (i.e., singular) at \pm 60 deg latitude (and negative for $|\varphi|>60$ deg), preventing the computation of $W_{S v H}$. This problem could be circumvented by using, e.g., $\delta=\Delta \lambda \cos \varphi$, but this is a coarse approximation in that it violates the rectangular-shaped cell requirement.

\subsubsection{Two-dimensional integration}

To more rigorously account for the [potentially] rectangular shape of $\Delta \varphi, \Delta \lambda$ grid cells, mean kernel values are computed by means of the 2D-AI, shown in Sect. 2.4. For the 2D-AI of mean Stokes kernels $\bar{S}(u)$, the planar approximation $K_{S}(x, y)$ of Stokes's kernel $S(\psi)$ is required. With the 2/ $\psi$ approximation [Eq. (19)] and planar approximation of the spherical distance $\psi \approx \sqrt{x^{2}+y^{2}}$, the planar Stokes kernel $K_{S}(x, y)$ reads: 


$$
K_{S}(x, y) \approx \frac{2}{\sqrt{x^{2}+y^{2}}}
$$

where $(x, y)$ are the planar coordinates of the evaluation point $Q$ relative to the computation point $P$, as introduced in Eq. (10). For a cell bounded by $v=\left(x_{1}, y_{1}, x_{2}, y_{2}\right)$, the mean kernel $\bar{K}_{S}(v)$ in planar approximation is computed using Eq. (13), to give:

$$
\begin{aligned}
\bar{K}_{S}(v) & =\frac{1}{\Delta x \Delta y} \int_{x=x_{1}}^{x_{2}} \int_{y=y_{1}}^{y_{2}} K_{S}(x, y) d x d y=\left.\left.\frac{1}{\Delta x \Delta y}|| F_{S}(x, y)\right|_{y_{1}} ^{y_{2}}\right|_{x_{1}} ^{x_{2}} \\
& =\frac{1}{\Delta x \Delta y}\left[F_{S}\left(x_{1}, y_{1}\right)-F_{S}\left(x_{2}, y_{1}\right)-F_{S}\left(x_{1}, y_{2}\right)+F_{S}\left(x_{2}, y_{2}\right)\right]
\end{aligned}
$$

where $\Delta x \Delta y$ is the surface area of the cell and $F_{S}(x, y)$ the antiderivative of the planar kernel $K_{s}(x, y)$. The antiderivative $F_{S}(x, y)$ is given by (de Min 1994):

$$
F_{S}(x, y)=2\left[x \ln \left(y+\sqrt{x^{2}+y^{2}}\right)+y \ln \left(x+\sqrt{x^{2}+y^{2}}\right)\right] .
$$

With the weighting factor $W=\bar{K}_{S}(v) / K_{S}(x, y)$ [Eq. (16)], point Stokes kernels $S(\psi)$ are converted to mean Stokes kernels $\bar{S}(u)$ by:

$$
\bar{S}(u)=W S(\psi)
$$

where the vector $u=\left(\lambda_{1}, \varphi_{1}, \lambda_{2}, \varphi_{2}\right)$ contains the coordinates of the cell corners. This transformation is possible because the first term in Stokes's kernel (Eq. 18) is dominant in the inner zone. Importantly, there is no need to analytically integrate the (complete) Stokes function to obtain analytical mean kernels.

\subsection{Numerical computation of mean kernels}

\subsubsection{Computation variants}

GLQ (Sect. 2.3 and Appendix A1) is used as the first numerical integration method to yield whole-of-the cell estimates $\bar{S}_{G L Q}$ of Stokes's kernel:

$$
\bar{S}_{G L Q}=\frac{1}{\sum w_{i} w_{j}} \sum_{i=1}^{n} \sum_{j=1}^{m} w_{i} w_{j} S\left(\psi\left(\varphi_{P}, \lambda_{P}, \varphi_{Q^{\prime} j}, \lambda_{Q^{\prime} i}\right)\right)
$$

with $n=m$ is the degree of the quadrature, $w_{i}, w_{j}$ are the Gaussian weights and $\left(\varphi_{Q^{\prime} i,} \lambda_{Q^{\prime} j}\right)$ are the zero-crossings of the Legendre polynomials, transformed to the integration domain $\left[\lambda_{1} \lambda_{2}\right] \times\left[\varphi_{1} \varphi_{2}\right]$. For the computation of the Gaussian weights $w_{i}, w_{j}$ and of the coordinates $\left(\varphi_{Q^{\prime} i}, \lambda_{Q^{\prime} j}\right)$ see Appendix A1. 
As a second method, a rectangular integration method, is included for comparison purposes. This method, called herein equidistant numerical integration (ENI), is based on equally spaced evaluation points. In comparison to GLQ, the kernel function values are not weighted. The spacing between adjacent evaluation points is $n^{-1} \Delta \lambda$ in the East-Westdirection ( $n^{-1} \Delta \varphi$ in the North-South-direction), and the distances of the outermost evaluation points from the cell boundary are $(2 n)^{-1} \Delta \lambda$ and $(2 n)^{-1} \Delta \varphi$, respectively. The arithmetic average of the $n^{2}$ point kernel values (Eq. 18), as computed at the equally spaced evaluation points, is used as numerical mean kernel estimate:

$\bar{S}_{E N I}=\frac{1}{n^{2}} \sum_{i=1}^{n} \sum_{j=1}^{n} S\left(\psi\left(\varphi_{P}, \lambda_{P}, \varphi_{Q^{\prime}}+\frac{2 i-n-1}{2 n} \Delta \varphi, \lambda_{Q^{\prime}}+\frac{2 j-n-1}{2 n} \Delta \lambda\right)\right), \mathrm{n} \geq 1$.

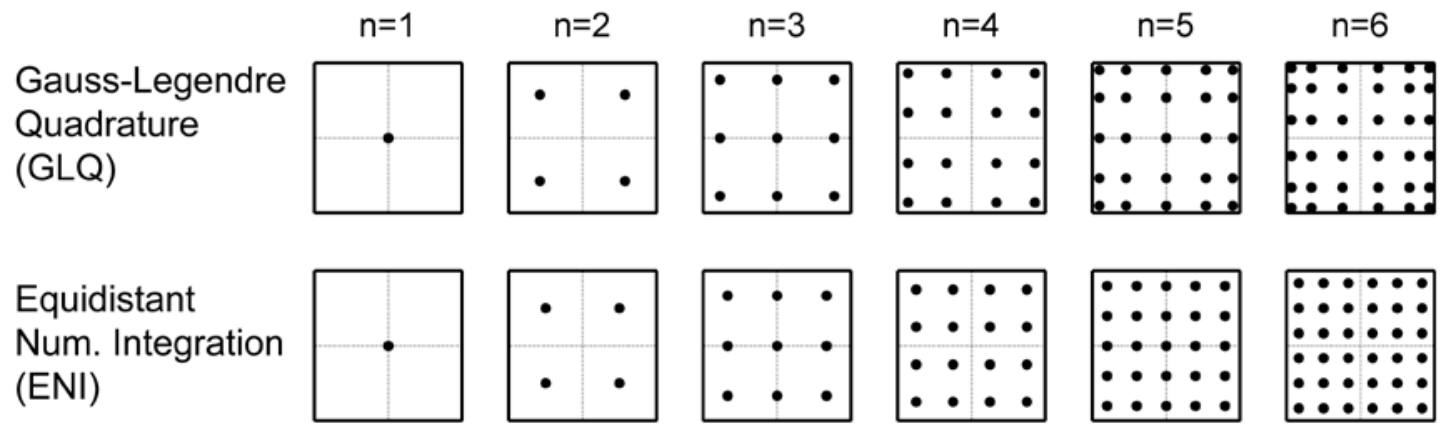

Fig 1 Arrangement of evaluation points across the cell as a function of parameter $n$. Top: GLQ. Bottom: ENI.

Figure 1 shows the arrangement of the GLQ and ENI evaluation points across the cell as a function of parameter $n$, ranging from 1 to 6 . Any cell shown represents the twodimensional integration domain $\left[\lambda_{1} \lambda_{2}\right] \times\left[\varphi_{1} \varphi_{2}\right]$. Both methods have in common that the evaluation points never coincide with the cell boundaries $\lambda_{1}, \lambda_{2}, \varphi_{1}, \varphi_{2}$. In case $n=1$, the evaluation point is located at the cell centre and both methods are identical, which is degeneration to the approximation of using just the centre-of-cell point kernel as mean kernel estimate. For $n \geq 2$, the GLQ point arrangement deviates from the equally spaced ENI evaluation points, showing the locations of the (transformed) zero-crossings of the $n$-th order Legendre polynomial (cf. Appendix A1).

To analyse the convergence behaviour of both methods, Eqs. (26) and (27) were evaluated as a function of $n$, ranging from 1 (centre-of-cell case) to 100, for a 1 arc-min cell in the direct vicinity of the innermost zone. Figure 2 shows the convergence error, given by

$\varepsilon=\left|\frac{\bar{S}_{G L Q / E N I}-\bar{S}_{G L Q}^{r e f}}{\bar{S}_{G L Q}^{r e f}}\right|$, 
i.e., the relative differences of the mean kernel estimates with respect to a 'reference' mean value $\bar{S}_{G L Q}^{r e f}$ obtained from GLQ for $n=1000$. As expected, the finer the subdivision of the cell with evaluation points, the better the approximation of the 'reference' mean kernel value through the numerical mean kernel estimate of both methods (Fig. 2).

GLQ offers substantially better convergence behaviour than ENI. For instance, with a total of $n^{2}=9$ evaluation points, GLQ mean kernel estimates fall below an arbitrary convergence threshold of $\varepsilon=10^{-4}$, while the ENI mean kernel estimates require about $n^{2} \approx$ 1000 evaluation points for a similar level of approximation. With $n^{2} \approx 100$ evaluation points, GLQ mean kernel estimates approximate the reference value better than $\varepsilon=10^{-10}$, [more than] good enough for any practical application of geodetic integrals, while the ENI method reaches only $\varepsilon=10^{-3}$. This convergence experiment shows the superiority of the GLQ method over (unweighted and) equally spaced evaluation points, as it is the case with ENI. Therefore, GLQ is recommended for the numerical mean kernel computation and will be used throughout the sequel.

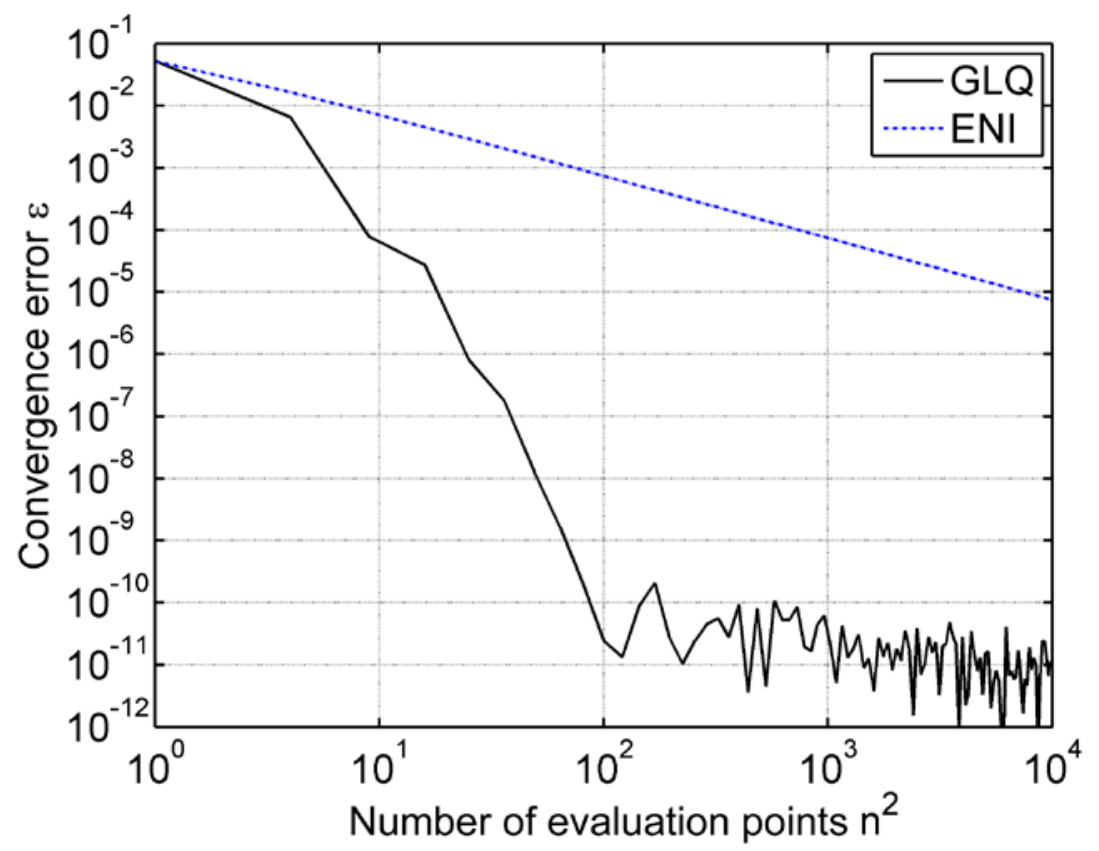

Fig 2 Convergence error $\varepsilon$ (Eq. 28) as a function of the number of evaluation points $n^{2}$. 'Reference' mean kernel value $=7279.97437550$ (from a degree $n=1000$ GLQ, i.e., $1,000,000$ evaluation points). This test cell is located 1 arc-min North of the innermost zone. Kernel $=$ Stokes's, grid resolution $=1$ arc-min, innermost zone latitude $=-35$ deg.

\subsubsection{Convergence behaviour}

For the practical application of GLQ numerical mean kernels, it is useful to define a convergence threshold. A simple estimation of a suitable convergence threshold is as follows: For remove-compute-restore (RCR) geoid computation, we assume that residual geoid undulation $N^{\text {res }}$, computed from residual gravity anomalies $\Delta g^{\text {res }}$, is $5 \mathrm{~m}$ or smaller. 
[Given that $N^{\text {res }}$ have a global RMS of $\sim 2.5 \mathrm{~m}$ when a degree-20 reference field is removed, this assumption will cover the extreme case of most practical geoid computations.] Then, a convergence error of $\varepsilon=10^{-4}$ for the mean kernel at any cell of a gravity grid would translate into a geoid undulation of $0.5 \mathrm{~mm}$ [Eq. (17)].

Depending on the spectral content of $\Delta g^{\text {res }}$ with a high-degree reference field removed (e.g., 360 or 2190), the $N^{\text {res }}$ will even be smaller, and so is the impact of the mean kernel convergence error on the geoid undulation. We therefore consider a convergence threshold of $\varepsilon=10^{-4}$ to be mostly acceptable for Stokes-based geoid computation, particularly when a high-degree reference field is removed. The same threshold was proposed by de Min (1994). In the extreme case where Stokes integration is used without removing a reference field contribution from the gravity anomalies, the global geoid signal is $\sim 30 \mathrm{~m}$ on average. This would require the use of a convergence threshold of $\varepsilon=10^{-5}$ (Sect. 3.5).

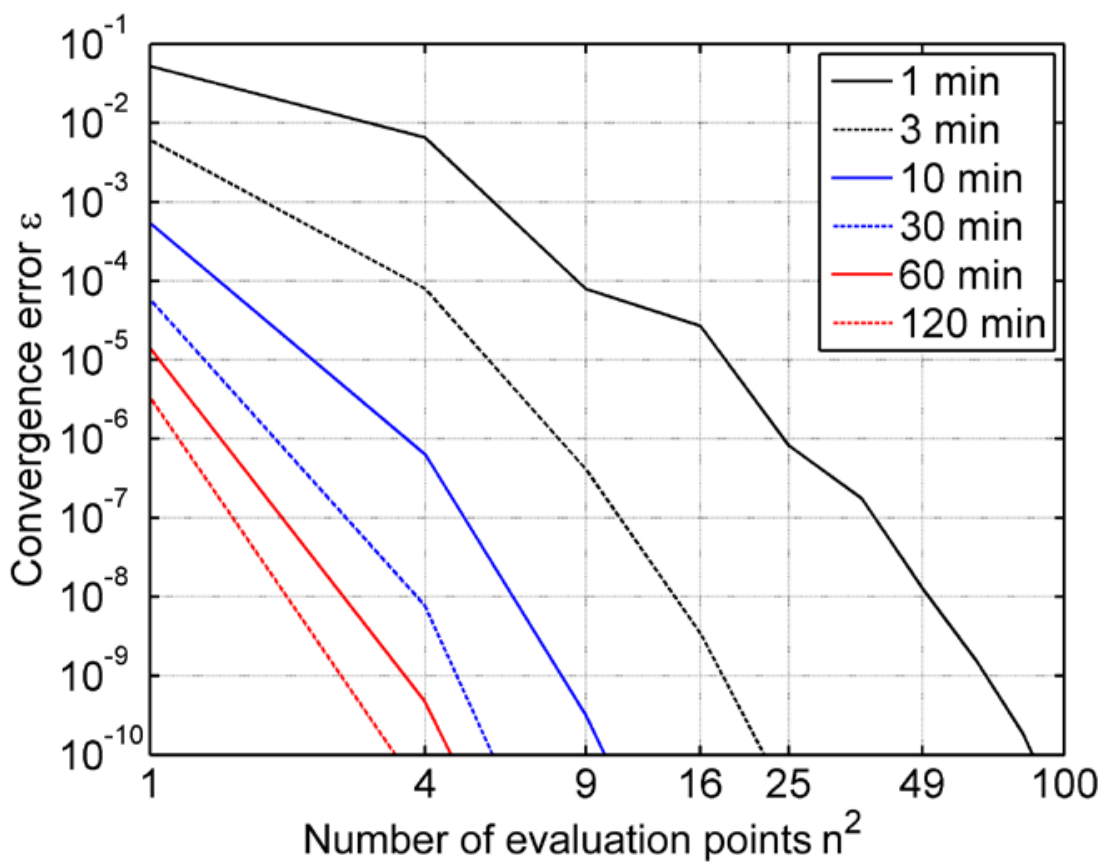

Fig 3 Convergence behaviour of GLQ numerical mean kernels. For cells located 1, 3, 10, 30, 60 and 120 arc-min North of the innermost zone, the convergence error between numerical mean kernel values and the reference value is shown as a function of the number of subcells used. The six reference values originate from GLQ quadrature degree $n=1000$. Kernel $=$ Stokes's, grid resolution $=1$ arc-min, innermost zone latitude $=-35$ deg.

The convergence behaviour of Stokes's GLQ numerical cell-mean values is further analysed as a function of the cell distance $\psi$ from the innermost zone and the number of evaluation points $n^{2}$ (Fig. 3). Depending on the spherical distance $\psi$ between the cell and innermost zone, different GLQ degrees $n$ are required for the estimates to fall below the threshold of $10^{-4}$ to $10^{-5}$. In the direct vicinity of the innermost zone, quadrature degrees $n=$ 3 to 5 (9..25 evaluation points) are required. At 10-30 arc-min from the innermost zone, $n=$ 2 is required per cell. At 30 arc-min and beyond, the centre-of-cell approximation (cf. Fig. 3, 
$n=1$ ) is good enough to meet the $10^{-4}$ criterion. Beyond 60 arc-min, the mean kernel approximation error is $\sim 10^{-5}$ when using centre-of-cell kernels. The convergence curves in Fig. 3 demonstrate that the computational requirements for GLQ numerical mean kernels are very low. Our experiment here can easily be replicated for other integral kernels (cf. Section 4).

\subsection{Comparison of the mean kernel computation methods}

Insight into the characteristics of mean kernels and the agreement between the two analytical solutions (Sect. 3.2) and the numerical solution (Sect. 3.3) is provided by a simple series of 2D kernel computations. For a regular 1 arc-min grid and an innermost zone latitude of $\varphi_{P}=$ $-35^{\circ}$, two-dimensional arrays of Stokes's kernel were prepared in terms of (a) point values, (b) mean values from the GLQ numerical approach [Eq. (26)] with $\varepsilon=10^{-5}$, (c) the $1 \mathrm{D}-\mathrm{AI}$ approach with $\delta=\Delta \lambda \cos \varphi$, [Eqs. (20) and 21)], and (d) the 2D-AI approach [Eqs. (22)-(25)].

The point kernel array is shown in Fig. 4a and the relative differences between the GLQ numerical mean and point kernel in Fig. 4b, expressed as a percentage. Figs. 4c and 4d show the relative differences between the 1D-AI and 2D-AI mean kernels and the GLQ mean kernels, which can be interpreted as approximation errors resulting from the analytical methods. The innermost zone contribution originates from the Haagmans et al. (1993, p. 240) solution and is thus the same for all kernel arrays.

From Fig. 4b, the largest differences of $~ 5-6 \%$ between mean and point kernels occur at the cells adjoining the innermost zone. Within a 2 arc-min radius, the differences are about $1-2 \%$ and quickly decrease to below $1 \%$ beyond. [The same results can also be deduced based on Fig. 3, from function values for $n^{2}=1$ ]. Furthermore, the 2D-AI and GLQ numerical solutions are in good agreement (Fig. 4d), while the 1D-AI solution deviates (Fig. 4c). This is particularly visible for cells along the central parallel where the 1D-AI results differ from the numerical and 2D-AI solution by as much as $\sim 9 \%$. However, this is not unexpected, recalling that the 1D-AI solution presumes square-shaped cells (Sect. 3.2.1), while the other mean kernel computation methods take into account the actual cell geometry.
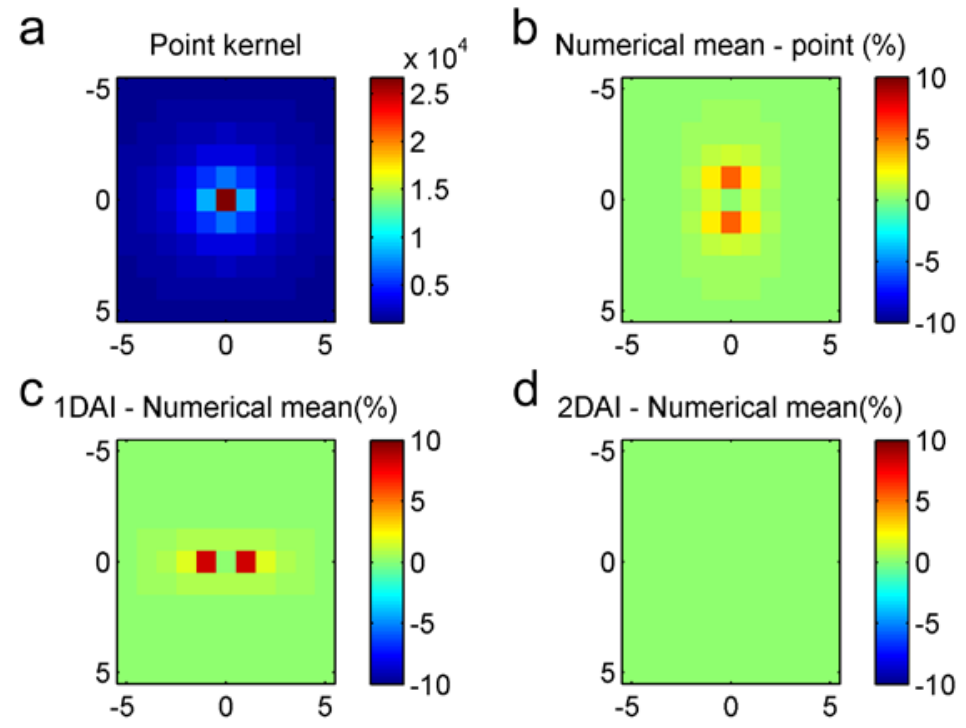
Fig 4 a: Point values of Stokes's kernel, b: differences between GLQ numerical mean and point kernels, c: differences between 1D-AI and GLQ mean, d: differences between 2D-AI and GLQ mean. Differences are given in percent. Each panel shows a 11 x 11 cell array of 1 arc min $x 1$ min cells, centred on the innermost zone. Parallel of innermost zone is -35 deg.

The percentage differences between the 2D-AI and GLQ numerical Stokes's mean kernels are shown for a larger area (61 x 61 arc-mins) with a different scaling in Fig. 5. The differences are largest near the innermost zone $\left(\sim 2 \times 10^{-4}\right)$, and are otherwise below or well below $10^{-4}$. Most likely, they reflect the approximation errors of the 2D-AI mean kernel computation. We consider this to be a satisfactory agreement between the two independent approaches.

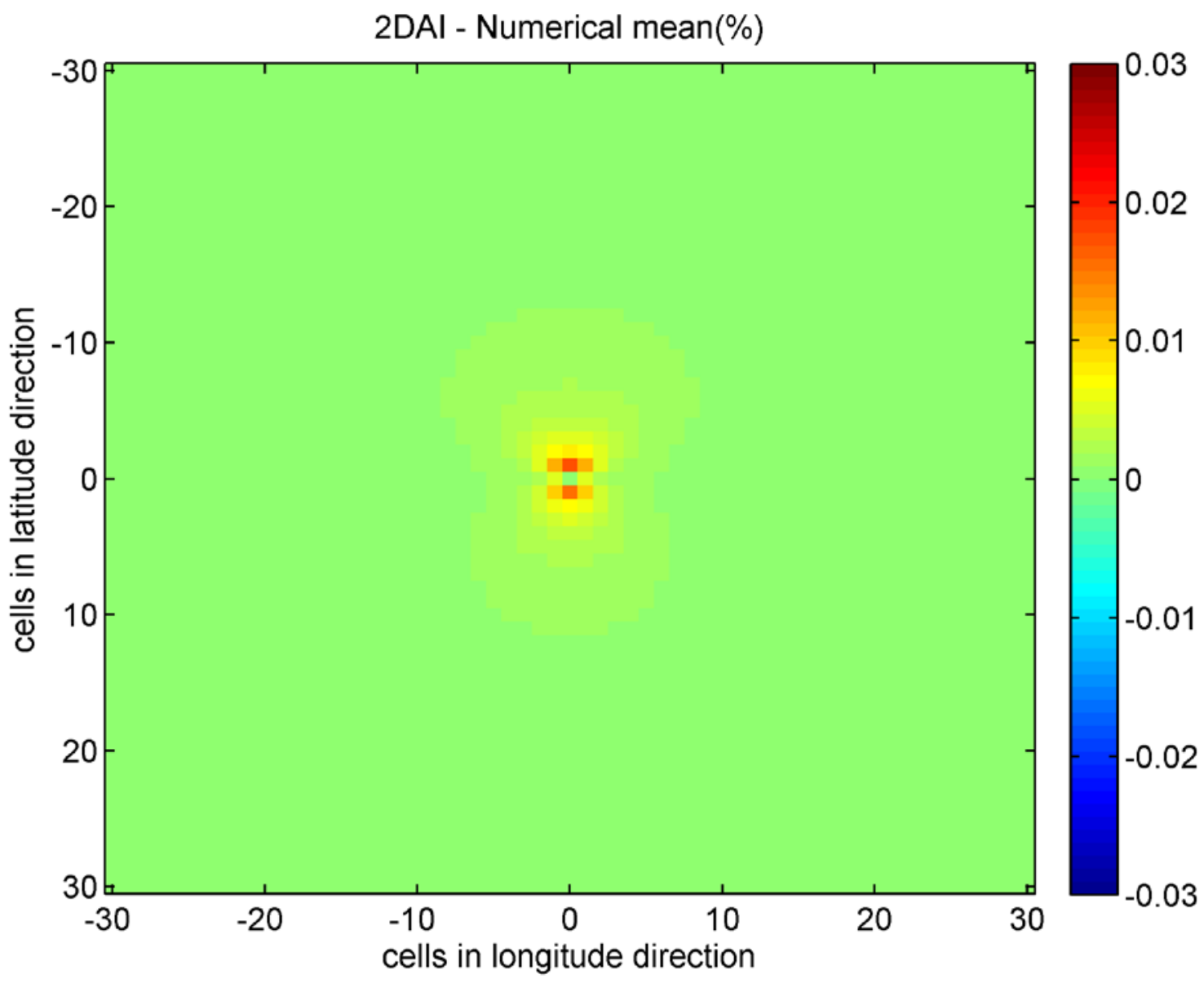

Fig 5 Percentage differences between 2D AI mean and numerical mean Stokes kernels for 61 $\mathrm{x} 61$ cells centred on the innermost zone. Grid resolution $=1$ arc min, central parallel $=-35$ deg.

Further insight into the characteristics of mean kernels is gained by a series of comparisons for various latitudes $\varphi_{P}$ of the central meridian ranging from $0^{\circ}$ to $70^{\circ}$. What we term the mean kernel effect (i.e., the differences between GLQ numerical mean and point 
kernels) is shown in Fig. 6 as a percentage. [The 2D-AI mean kernels exhibit a very similar behaviour so are not depicted]. The differences gradually change from patterns with equal symmetry in North-South and East-West directions at the equator to patterns that exhibit two symmetry directions at high latitudes. This indicates that the point kernel approximation will also introduce numerical integration errors that increase with latitude.
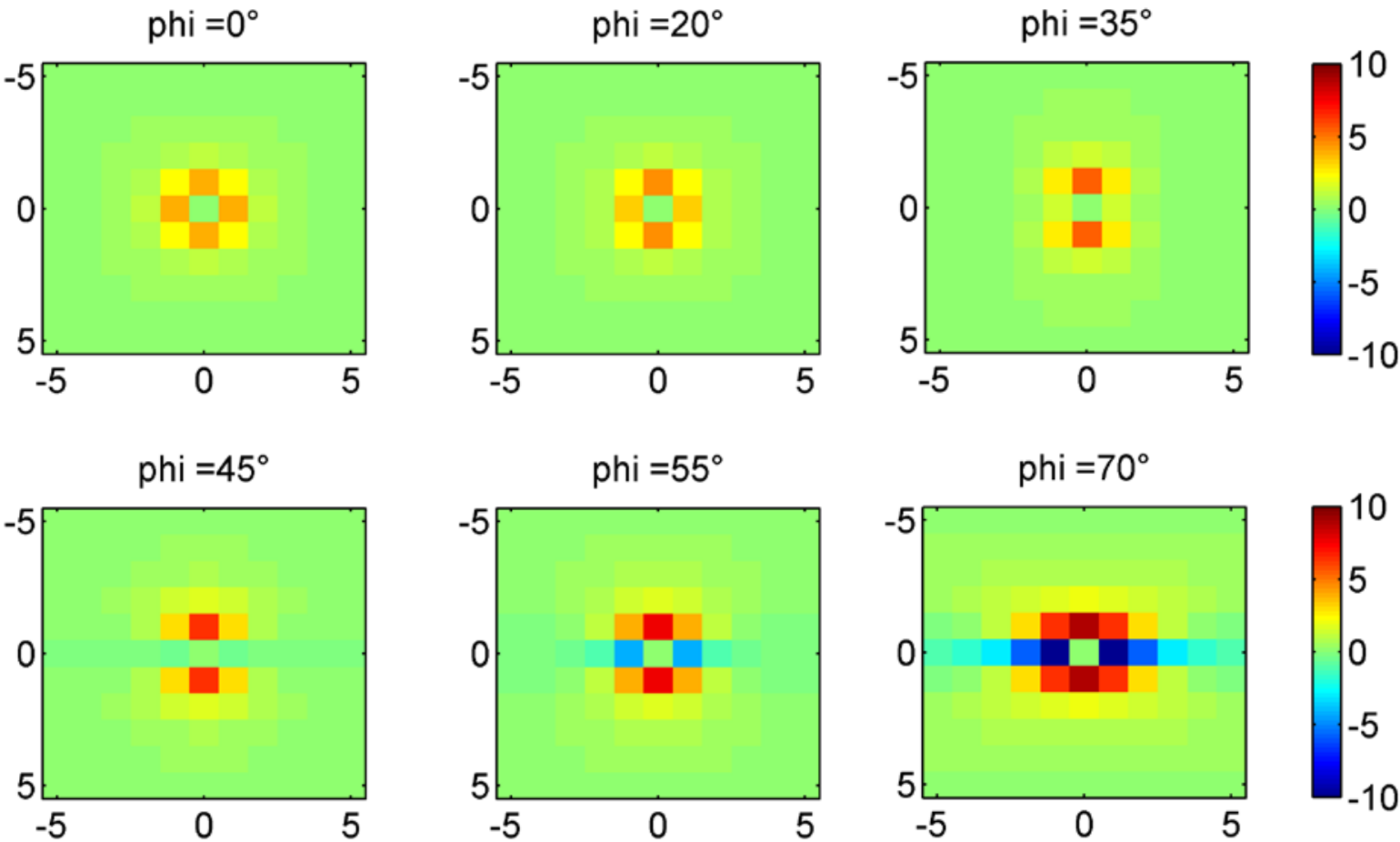

Fig 6 Percentage differences between the numerical mean and point kernels with latitude $\left(0^{\circ}\right.$, $20^{\circ}, 35^{\circ}, 45^{\circ}, 55^{\circ}$ and $70^{\circ}$ ). Each panel shows $11 \times 11$ kernel arrays centred on the innermost zone. For latitude $=70^{\circ}$, the maximum differences are $-16.4 \%$ (the cells adjoining the innermost zone). Cell size $=1$ arc $\min \times 1$ arc min, kernel $=$ Stokes.
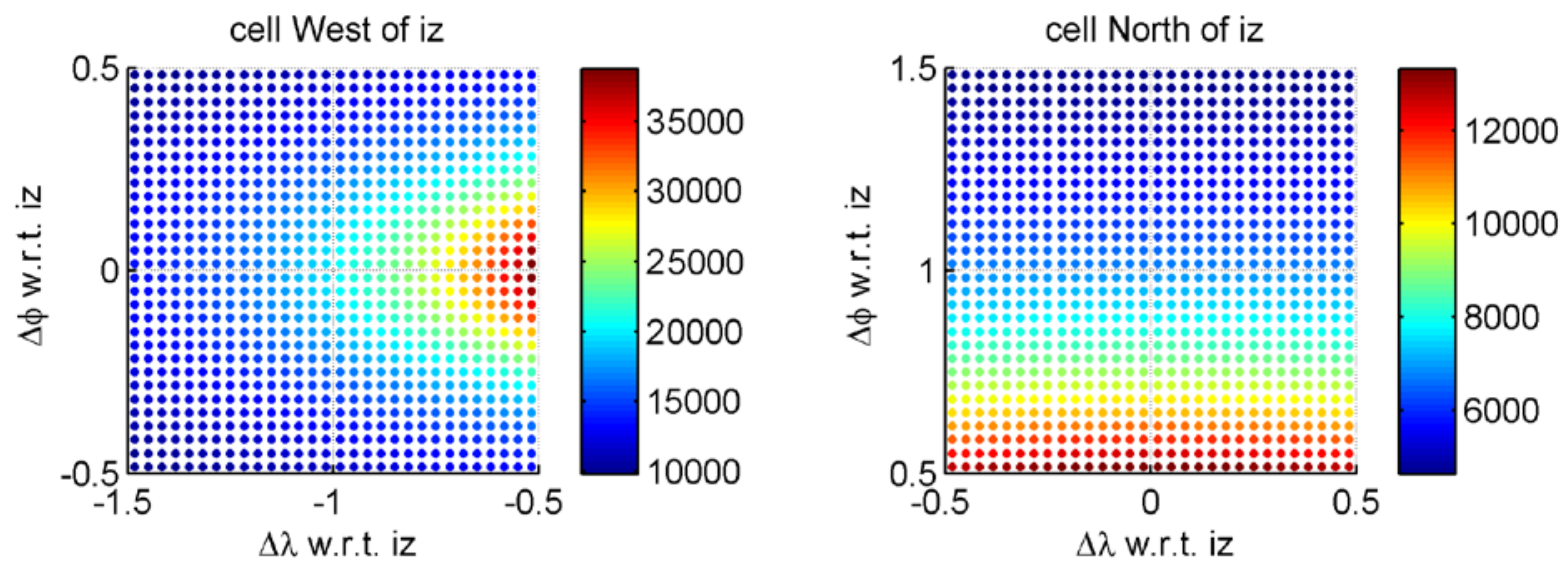

Fig 7 Detailed results of the numerical mean kernel for $70^{\circ}$ latitude. Left: Cell adjoining the innermost zone in the Western direction. Right: Cell adjoining the innermost zone in the 
Northern direction. Note: Both cells have a pronounced rectangular shape due to meridional convergence. Cell size $=1$ arc $\min x 1$ arc min.

The mean kernel values are not always larger than point kernel values. Higher than $\sim 43^{\circ}$ in latitude, negative values occur in the central parallel direction, which become more pronounced towards the poles. At $70^{\circ}$ latitude, the differences are as large as $-16.4 \%$. The reason for these negative differences is meridional convergence affecting the cell geometry, which is further explained by Fig. 7, showing dense grids of point kernel values over two cells adjacent to the innermost zone.

For the cell West of the innermost zone (Fig. 7, left), the mean kernel is $16.4 \%$ smaller than the point value at the cell centre. For the cell North of the innermost zone (Fig. 7 , right), the mean kernel is $9 \%$ larger than the point kernel. The behaviour of mean kernels visible in Figs. 6 and 7 demonstrates that the 1D-AI approach by Strang van Hees (1990) is of less use to model the complex 2D difference patterns that occur between mean and point kernels at some distance from the equator. Difference patterns very similar to those shown in Fig. 7 are found for other grid resolutions, e.g. 0.1 arc min and 10 arc min.

\subsection{Numerical closed-loop tests}

To assess the performance of the mean kernel approaches presented in Sects 3.2 and 3.3, closed-loop tests were performed based on the EGM2008 (Pavlis et al. 2008) global geopotential model (GGM). The idea behind a closed-loop test is to use self-consistent pairs of gravity $\Delta g^{G G M}$ and geoid heights $N^{G G M}$, which are derived from the same disturbing potential $T$ (see e.g., Torge 2001, pp 215, 271 and 272 for the equations), for practical testing of Stokes’s integration (cf. Tziavos 1996; Novák et al. 2001; Featherstone 2002).

We assume that the $\Delta g^{G G M}, N^{G G M}$ pairs are self-consistent, in that, (hypothetically) error-free numerical evaluation of Stokes's convolution integral would exactly reconstitute the geoid height $N^{G G M}$ from the gravity anomalies $\Delta g^{G G M}$. Naturally, any errors in the numerical evaluation of Stokes's integral [e.g., due to the kernel type (point or mean values), discretisation of gravity anomalies, errors in the software implementation, limited computational precision, etc...] will cause differences between $N^{G G M}$ and $N\left(\Delta g^{G G M}\right)$ from Eq. (17). The main advantage of such a closed-loop test is that the results are not 'swamped' by noisy comparison data (e.g., observed GPS/levelling and gravity data). As a disadvantage, the GGM-implied $\Delta g^{G G M}$ and $N^{G G M}$ data is always band-limited due to the limited model resolution (maximum degree $M$ ), as such only capable of approximating the 'real' terrestrial gravity field to some extent. With EGM2008, however, a GGM is now available that very closely approximates Earth's gravity field over most regions (e.g., Newton's Bulletin 2009).

The sole parameter varied in our closed-loop tests is the mean kernel computation strategy, in the following ways: (i) point kernel, (ii) GLQ numerical mean $\left(\sim 10^{-5}\right)$, (iii) $1 \mathrm{D}-\mathrm{AI}$ mean, and (iv) 2D-AI mean. Our closed-loop tests use EGM2008 on a global 10 arc-min grid $(1080 \times 2160$ cells $)$ within the spectral band $2 \ldots 360$. The 10 arc-min grid resolution was 
selected such that the shortest wavelength components of the degree $21 . . .360$ truncation are not under-sampled. For the harmonic synthesis of $\Delta g^{G G M}$ and $N^{G G M}$, we used a slightly altered version of harmonic_synth.f (Holmes and Pavlis 2008). The synthesis was performed in full spherical approximation, i.e., at the surface of a sphere with constant radius $R=$ $6378137 \mathrm{~m}$, and in terms of coordinate grids equally spaced in terms of geocentric latitude. The same constant radius was used in the numerical evaluation of Stokes's integral [Eq. (17)]. As a consequence, both the harmonic synthesis and the numerical integration are based on the same level of spherical approximation.

For the numerical evaluation of Stokes's integral, we use the in-house software FFT1Dmod2010 at the Western Australian Centre for Geodesy, which makes use of the 1D FFT approach (Haagmans et al. 1993). A predecessor version was used for the computations of AUSGeoid09 (Featherstone et al. 2010) and NZGEOID09 (Claessens et al. 2011). The computation approaches described in Sects. 3.2 and 3.3 were implemented in FFT1Dmod2010, allowing inter-comparisons between the mean kernel variants. For the practical computations, we used the iVEC high-performance computational facility (http://www.ivec.org/), allowing us to accelerate the numerical tests.

Table 1 reports the descriptive statistics from the closed-loop tests. The differences using point kernels and GLQ mean kernels are shown in Fig. 8 (the results using 2D-AI are very similar so not shown). The point kernel shows a global RMS of $\pm 67 \mathrm{~mm}$ (Fig. 8a), while the GLQ numerical mean and 2D-AI mean kernels exhibit the lowest global RMS values of about $\pm 14 \mathrm{~mm}$ (cf. Fig. 8b). The equivalent performance of the numerical mean and 2D-AI mean kernels provides some evidence of the correctness of the results, and confirms indirectly the validity of Eq. (15). From Table 1, it follows that the 1D-AI mean kernel breaks down for irregular (rectangular) cell geometries. In conclusion, the numerical tests demonstrate the benefits of using mean kernels from 2D-AI or GLQ numerical integration.

Table 1 Descriptive statistics of closed-loop geoid differences $N^{G G M}-N\left(\overline{\Delta g}^{G G M}\right)$ for the global test area $-89.9167^{\circ} \leq \varphi \leq 89.9167^{\circ}$ and $-179.9167^{\circ} \leq \lambda \leq 179.9167^{\circ}(1,080 \times 2,160=$ 2,332,800 points, 10 arc-min resolution, EGM2008 2..360 truncation, $N^{G G M} \mathrm{~min} / \mathrm{max} /$ mean/ RMS: - $-106.47 /$ 86.65/ -0.81 / 28.99 m ). Units in m.

\begin{tabular}{|l|c|c|c|c|}
\hline Kernel type & Min & Max & Mean & RMS \\
\hline Point ( centre-of-cell) & -0.477 & 0.890 & 0.005 & 0.069 \\
\hline $10^{-5}$ GLQ numerical mean & -0.230 & 0.383 & 0.000 & 0.014 \\
\hline 1D-AI mean & -0.336 & 0.911 & 0.005 & 0.070 \\
\hline 2D-AI mean & -0.221 & 0.433 & 0.000 & 0.014 \\
\hline
\end{tabular}



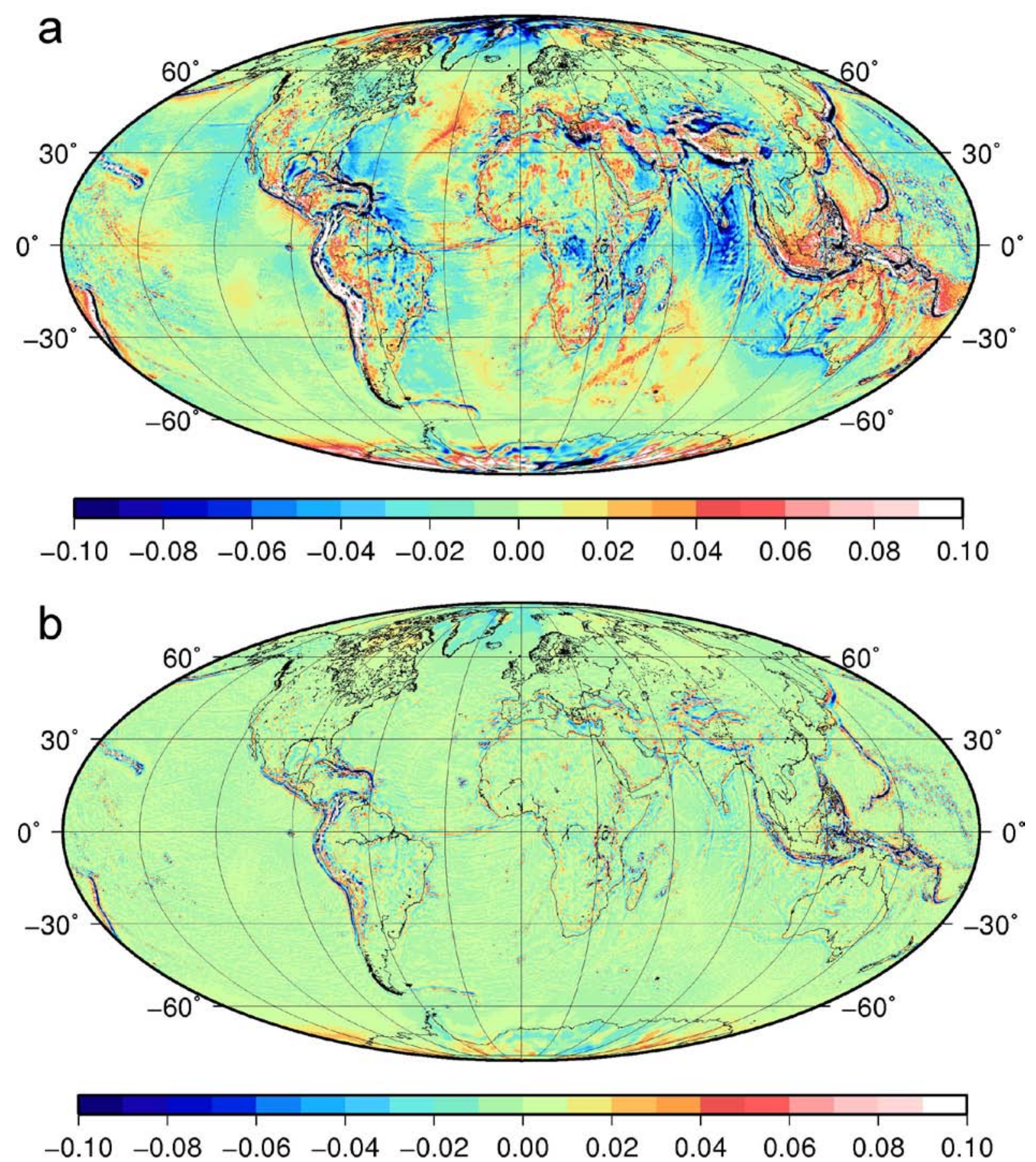

Fig 8 Closed-loop $N^{G G M}-N\left(\Delta g^{G G M}\right)$ geoid differences. a) results of Stokes's integration using point kernels, b) results of Stokes's integration using GLQ mean kernels. Mollweide projection (centred to $0^{\circ}$ longitude, meridians $30^{\circ}$ apart), Units in metres. 


\section{Mean kernel computation for other geodetic integrals}

This section attempts to provide a compendium of analytical solutions to the computation of mean kernels for a variety of other geodetic integrals. The grouping is neither done according to the techniques used to collect the observations, nor in terms of the target quantity. Instead, we arrange the integrals that are represented by similar or identical functions in the inner zone. This is pragmatic because the approximations are identical or similar, and so are the antiderivatives $F(x, y)$. The antiderivatives $F(x, y)$ are given in Sect. 4.7, which are the key for the analytical mean kernel computation as stated in Sect. 2 and shown in Sect. 3.

We follow the conventions used in Sect. 2. Quantities with over-bars denote mean values of the cell, $\psi$ is the spherical distance between the computation point $P$, and the data point $Q$ under evaluation. The rectangular coordinates $(x, y)$ are the planar coordinates of the evaluation point $Q$ relative to computation point $P$ [Eq. (10)]. Vector $u=\left(\lambda_{1}, \varphi_{1}, \lambda_{2}, \varphi_{2}\right)$ denotes the cell boundaries in terms of spherical coordinates and $v=\left(x_{1}, y_{1}, x_{2}, y_{2}\right)$ denotes the cell boundaries in planar coordinates.

We acknowledge that the deflection-geoid (Sect 4.2) and Vening-Meinesz and inverse Vening-Meinesz integrals (Sect 4.3) are not strictly defined as convolution integrals, because they are dependent on azimuth $\alpha$. Nonetheless, these integrals can be accurately evaluated with the analytical and numerical methods described in this paper.

\subsection{Stokes-type integrals}

These kernels have similar asymptotic representations for small values of $\psi$, which is $c / \psi$, where $c$ denotes a constant that may be different for the individual integrals (cf. Table 2).

\subsubsection{Hotine’s integral}

Hotine's integral (Hotine 1969, p311; also see Zhang 1993) establishes the relation between the geoid undulation $N$ and gravity disturbances $\delta g$ :

$$
N=\frac{R}{4 \pi \gamma} \iint_{\sigma} A(\psi) \delta g d \sigma
$$

where

$$
A(\psi)=\frac{1}{\sin (\psi / 2)}-\ln \left(1+\frac{1}{\sin (\psi / 2)}\right)
$$

Because the first term in Stokes's integral $S^{1 s t}(\psi)$ is the dominant term near the computation point $P$, the planar approximation $K_{A}(x, y)$ of Hotine's kernel is identical with that of Stokes's kernel [Eq. (22)]: i.e., $K_{A}(x, y)=K_{S}(x, y)$. 


\subsubsection{Eötvös's integral}

Eötvös’s integral (e.g., Zhang 1995) describes the relationship between the gravity anomaly $\Delta g$ and the vertical gradient of the disturbing potential $T_{r r}$ :

$$
\Delta g=\frac{R}{4 \pi} \iint_{\sigma} B(\psi) T_{r r} d \sigma
$$

where

$$
B(\psi)=\frac{1}{\sin (\psi / 2)}+9-18 \sin (\psi / 2)+(2-9 \cos \psi) \ln \left(1+\frac{1}{\sin ^{2}(\psi / 2)}\right)
$$

Again, the planar approximation of Eötvös's kernel $K_{B}(x, y)$ is the same as Stokes's kernel: $K_{B}(x, y)=K_{S}(x, y)$.

\subsubsection{Green-Molodensky integral}

The Green-Molodensky integral is used to compute surface density $\rho$ from gravity anomalies $\Delta g$ (Hotine 1969, p346):

$$
\rho=\frac{1}{16 \pi^{2}} \iint_{\sigma} G(\psi) \Delta g d \sigma
$$

where

$$
\begin{aligned}
G(\psi)= & 1+\frac{3}{\sin (\psi / 2)}-18 \sin (\psi / 2)-21 \cos (\psi) \\
& -9 \cos \psi \ln \left[\sin (\psi / 2)+\sin ^{2}(\psi / 2)\right]
\end{aligned}
$$

This kernel can be approximated by $6 / \psi$ near the innermost zone. In planar approximation, the Green-Molodensky kernel reads: $K_{G}(x, y)=3 K_{s}(x, y)$.

\subsubsection{Tidal displacement integral}

This establishes the relation between ocean tide height $Z$ and a change in geoid undulation $\delta N$ that can be computed using (Vaníček and Krakiwsky 1986, p602):

$$
\delta N \approx-\frac{G \rho_{W} R}{\sqrt{2} g} \iint_{\sigma} L(\psi) Z d \sigma
$$

where $G$ is the Newtonian gravitational constant, $\rho_{W}$ the density of water and $g$ gravity, and

$$
L(\psi)=(1-\cos \psi)^{-1 / 2}
$$

A planar approximation is derived using the approximation $1-\cos \psi \approx 0.5 \psi^{2}$ for small $\psi$. 


$$
K_{L}(x, y) \approx \frac{1}{\sqrt{0.5}} \frac{1}{\sqrt{x^{2}+y^{2}}}=\frac{1}{\sqrt{2}} K_{S}(x, y) .
$$

The planar approximation $K_{L}(x, y)$ differs from Stokes's $K_{S}(x, y)$ by a constant factor of $1 /(\sqrt{2})$.

\subsubsection{Ocean tide loading integral}

According to Bos and Baker (2005), the gravity ocean tide loading (OTL) effect $\delta g_{\text {отL }}$ can be computed using:

$$
\delta g_{\text {ОтL }}=\frac{g}{M} \iint_{\sigma} \rho_{W} F(\psi) Z d \sigma
$$

where $g$ is average gravity at the Earth's surface, $M$ is mass of the Earth, $\sigma_{w}$ is density of water, $\mathrm{Z}$ is the ocean tide (a complex number). The kernel $F(\psi)$ is the gravity Green's function:

$$
F(\psi)=\sum_{n=0}^{\infty}\left(n+2 h_{n}^{\prime}-(n+2) k_{n}^{\prime}\right) P_{n}(\cos \psi)
$$

where $h_{n}^{\prime}, k_{n}^{\prime}$ are the load Love numbers of degree $n$ and $P_{n}(\cos \psi)$ is the Legendre polynomial of degree $n$. Those parts of $F(\psi)$ that are independent of the load Love numbers can be expressed - after a correction accounting for the height of evaluation - in closed-form as (Bos and Baker 2005):

$$
F^{\prime}(\psi)=-\frac{2 \sin ^{2}(\psi / 2)+p}{\left[4 \sin ^{2}(\psi / 2)(1+p)+p^{2}\right]^{3 / 2}}
$$

with $p=h / R, h$ is the height of evaluation. A planar approximation $K_{F^{\prime}}(x, y)$ for $F^{\prime}(\psi)$ is found for the case $h=0$, where Eq. (40) simplifies to $F^{\prime}(\psi) \approx-0.5 / \psi$ near the computation point, allowing the analytical computation of mean kernel values (cf. Sect. 4.7). The planar approximation $K_{F^{\prime}}(x, y)$ is related to the one for Stokes's function by: $K_{F^{\prime}}(x, y)=$ $-0.25 K_{S}(x, y)$.

For the case $h \neq 0$, the planar approximation of Eq. (40) is not straightforward. However, the computation of mean kernel estimates is still possible by applying the numerical integration method described in Sect 2.3.

\subsection{Deflection-geoid integral}


This can be used for computation of geoid undulations $N$ from vertical deflections $(\xi, \eta)$, which are typically available from satellite radar altimetry. The deflection-geoid integral reads (Hwang 1998, p307):

$$
N=\frac{R}{4 \pi} \iint_{\sigma} C^{\prime}(\psi)(\xi \cos \alpha+\eta \sin \alpha) d \sigma
$$

where

$$
C^{\prime}(\psi)=-\cot (\psi / 2)+3 / 2 \sin (\psi) .
$$

and the quantity $\alpha$ denotes the azimuth from the computation point to the data points. The kernel $C^{\prime}(\psi)$ is approximated by $-2 / \psi$ in the inner zone. In planar approximation, Eq. (41) reads:

$$
N=\frac{1}{4 \pi} \iint_{\sigma} K_{C^{\prime}}^{\xi}(x, y) \xi+K_{C^{\prime}}^{\eta}(x, y) \eta d x d y
$$

with the planar kernel elements

$$
\left\{\begin{array}{l}
K_{C^{\prime}}^{\xi}(x, y) \\
K_{C^{\prime}}^{\eta}(x, y)
\end{array}\right\}=\frac{-2}{\left(x^{2}+y^{2}\right)}\left\{\begin{array}{l}
y \\
x
\end{array}\right\} .
$$

\subsection{Vening-Meinesz-type integrals}

\subsubsection{Vening-Meinesz's integral}

This describes the computation of vertical deflections $(\xi, \eta)$ from gravity anomalies $\Delta g$ (Vening-Meinesz 1928, Heiskanen and Moritz 1967, p114, Torge 2001, p283):

$$
\left\{\begin{array}{l}
\xi \\
\eta
\end{array}\right\}=\frac{1}{4 \pi \gamma} \iint_{\sigma} V(\psi)\left\{\begin{array}{l}
\cos \alpha \\
\sin \alpha
\end{array}\right\} \Delta g d \sigma
$$

where

$$
\begin{aligned}
V(\psi)=\frac{\partial S(\psi)}{\partial \psi}=- & \frac{\cos (\psi / 2)}{2 \sin ^{2}(\psi / 2)}+8 \sin \psi-6 \cos (\psi / 2) \\
& -3 \frac{1-\sin (\psi / 2)}{\sin \psi}+3 \sin \psi \ln \left[\sin (\psi / 2)+\sin ^{2}(\psi / 2)\right]
\end{aligned}
$$

which is the derivative of Stokes's kernel $S(\psi)$ with respect to $\psi$. The planar approximation of $V(\psi)$ is (Schwarz et al. 1990, p496, El Habiby 2007): 


$$
\left\{\begin{array}{l}
\xi \\
\eta
\end{array}\right\}=\frac{1}{4 \pi \gamma} \iint_{\sigma}\left\{\begin{array}{c}
K_{V}^{\xi}(x, y) \\
K_{V}^{\eta}(x, y)
\end{array}\right\} \Delta g d x d y
$$

where the elements of the planar kernels read:

$$
\left\{\begin{array}{l}
K_{V}^{\xi}(x, y) \\
K_{V}^{\eta}(x, y)
\end{array}\right\}=\frac{-2}{\left(x^{2}+y^{2}\right)^{3 / 2}}\left\{\begin{array}{c}
y \\
x
\end{array}\right\}
$$

\subsubsection{Inverse Vening-Meinesz integral}

This converts vertical deflections $(\xi, \eta)$ to gravity anomalies $\Delta g$ (e.g., Hwang 1998, p306):

$$
\Delta g=\frac{\gamma}{4 \pi} \iint_{\sigma} H^{\prime}(\psi)(\xi \cos \alpha+\eta \sin \alpha) d \sigma
$$

where

$$
H^{\prime}(\psi)=-\frac{\cos (\psi / 2)}{2 \sin ^{2}(\psi / 2)}+\frac{\cos (\psi / 2)[3+2 \sin (\psi / 2)]}{2 \sin (\psi / 2)[1+\sin (\psi / 2)]}
$$

The $V(\psi)$ and $H^{\prime}(\psi)$ kernels have the same asymptotic representation of $-2 / \psi^{2}$ in the inner zone. In planar approximation, the inverse Vening-Meinesz formula reads:

$$
\Delta g=\frac{\gamma}{4 \pi} \iint_{\sigma} K_{H^{\prime}}^{\xi}(x, y) \xi+K_{H^{\prime}}^{\eta}(x, y) \eta d x d y
$$

The planar kernels of the Vening-Meinesz and inverse Vening-Meinesz integral are identical: $K_{H^{\prime}}^{\xi}(x, y)=K_{V}^{\xi}(x, y)$ and $K_{H^{\prime}}^{\eta}(x, y)=K_{V}^{\eta}(x, y)$.

\subsection{Inverse Stokes-type integrals}

\subsubsection{Inverse Stokes integral}

The inverse Stokes integral (also called Molodensky’s integral) yields gravity anomalies $\Delta g$ from geoid undulations $N$ (Molodensky et al. 1962 p341, Zhang 1993, p43, Torge 2001, p285):

$$
\Delta g=-\frac{\gamma}{R} N_{P}-\frac{\gamma}{16 \pi R} \iint_{\sigma} E(\psi)\left(N-N_{P}\right) d \sigma
$$

with $N_{P}$ the geoid undulation of the computation point and $N$ geoid undulation of the data points. The Molodensky kernel reads in closed-form:

$$
E(\psi)=\frac{1}{\sin ^{3}(\psi / 2)}
$$


Using approximate relations [Eqs. (19) and (22)], the planar approximation $K_{E}(\psi)$ is:

$$
K_{E}(x, y)=8\left(x^{2}+y^{2}\right)^{-3 / 2}
$$

\subsubsection{Inverse Hotine integral}

A similar integral is the inverse Hotine integral (also called the modified Molodensky integral) that converts geoid undulations $N$ to gravity disturbances $\delta g$ (Zhang 1993, p43):

$$
\delta g=+\frac{\gamma}{R} N_{P}-\frac{\gamma}{16 \pi R} \iint_{\sigma} E(\psi)\left(N-N_{P}\right) d \sigma
$$

For the kernel $E(\psi)$ and its planarisation $K_{E}(x, y)$, see Eqs. (53) and (54).

\subsection{Terrain-related geodetic integrals}

Three important representatives of terrain effect integrals are the gravimetric terrain correction $c_{T C}$, the primary indirect effect on the geoid $N_{\text {ind }}$ and Molodensky's $\mathrm{G}_{1}$ term. These integrals are evaluated by means of digital elevation data.

\subsubsection{Terrain correction integral}

The planar approximation of the gravimetric terrain correction $c_{T C}$ reads (e.g., Forsberg 1984; Forsberg 1985; Torge 2001, p265, Schwarz et al. 1990):

$$
c_{T C}=\frac{1}{2} G \rho \iint_{\sigma} T(l)\left(H-H_{P}\right)^{2} d x d y
$$

where $\rho$ is the [often-assumed constant] mass-density of the topography, $H_{P}$ is the terrain height of the computation point, $H$ the terrain height of the data points, and:

$$
T(l)=\frac{1}{l^{3}}=\left(x^{2}+y^{2}+z^{2}\right)^{-3 / 2} .
$$

In less elevated terrain, $T(l)$ is approximated linearly by (Schwarz et al. 1990, Torge 2001, p265):

$$
K_{T}(x, y)=\left(x^{2}+y^{2}\right)^{-3 / 2} \text {. }
$$

If the 3D separation between the computation and functional points is used in mountainous terrain [Eq. (57) instead of Eq. (58)], the analytical solution of the kernel is obtained in analogy to that of the planar Poisson integral kernel (Eq. 64).

\subsubsection{Primary indirect effect integral}


The indirect effect of Helmert's second condensation reduction on the geoid can be expressed in planar approximation by (e.g., Vaníček and Kleusberg 1987; Sideris and She 1995):

$$
N_{\text {ind }}=-\frac{\pi G \rho}{\gamma} H_{P}^{2}-\frac{G \rho}{6 \gamma} \iint_{\sigma} T(l)\left(H^{3}-H_{P}^{3}\right) d x d y
$$

where the kernel is the same as in Eq. (56).

\subsubsection{Molodensky's $\mathrm{G}_{1}$ term integral}

In the computation of quasigeoid heights $\zeta$ via Stokes's formula from gravity anomalies $\Delta g$, a correction called Molodensky's $\mathrm{G}_{1}$-term is applied to the gravity anomalies $\Delta g$ (Torge 2001, p 290):

$$
\zeta=\frac{R}{4 \pi \gamma} \iint_{\sigma}\left(\Delta g+G_{1}\right) \cdot S(\psi) d \sigma .
$$

Molodensky’s $\mathrm{G}_{1}$-term is computed from (Heiskanen and Moritz 1967, p 310):

$$
G_{1}=\frac{R^{2}}{2 \pi} \iint_{\sigma} T\left(l_{0}\right)\left(H-H_{P}\right) \Delta g d x d y
$$

where the kernel function $T$ given in Eq. (57) with $l_{0}=2 R \sin (\psi / 2)$. Hence, the planar approximations are also identical. It is acknowledged that other representations of the $G_{1}$ term exist (e.g., Moritz 1980; Val'ko et al. 2008). However, because the integral kernel of those other representations is the same as in Eq. (62), mean integral kernels can be obtained in complete analogy to the previous terrain effect integrals. The same holds for the Molodensky $G_{2}$-correction term, which is given, e.g., by Val'ko et al. (2008).

\subsection{Poisson integral}

Poisson's integral is used for the upward continuation of gravity given on a boundary surface. The spherical approximation reads (Hotine 1969, p323, Torge 2001, p293, El Habiby 2007):

$$
\Delta g_{P}=\frac{R^{2}\left(r^{2}-R^{2}\right)}{4 \pi r} \iint_{\sigma} T(l) \Delta g d \sigma
$$

where $\Delta g_{P}$ is the upward-continued gravity anomaly at point $P, \Delta g$ are gravity anomalies on the boundary surface, $r$ is the distance between centre of sphere $\mathrm{R}$ and point $P$ outside the sphere. The planar approximation of Eq. (62) is (Bláha et al. 1996):

$$
\Delta g_{P}=\frac{1}{2 \pi} z_{0} \iint_{\sigma} K_{D}(x, y) \Delta g d x d y
$$


with

$$
K_{D}(x, y)=\left(x^{2}+y^{2}+z_{0}^{2}\right)^{-3 / 2}
$$

and constant $z_{0}$ denoting elevation (e.g., flight height).

\subsection{Antiderivatives: discussion and examples}

For any of the geodetic convolution integrals described above, Table 2 summarises the respective asymptotic representations, the elements of the planar kernel $K(x, y)$ and their antiderivatives $F(x, y)$. Table 2 provides the information necessary to analytically compute mean kernel estimates for the accurate evaluation of the integrals in the inner zone. The antiderivative for the planar Stokes kernel has been published by de Min (1994, p42) and for the terrain correction by Forsberg (1984, p49).

Following the general concept in Sect. 2 and the example given in Sect. 3, analytical mean kernel computations are three-step procedures:

1. The planar kernel $K(x, y)$ is analytically integrated using the antiderivative $F(x, y)$ over a given cell bounded by coordinates $v=\left(x_{1}, y_{1}, x_{2}, y_{2}\right)$. This gives mean values of the kernel $\bar{K}(v)$ in planar approximation.

2. The planar kernel is evaluated at the centre of the cell, giving point kernel values $K(x, y)$ in planar approximation.

3. Kernel weighting factors $\bar{K}(v) / K(x, y)$ are applied to convert the point kernels to mean kernels.

For terrain effect kernels (which are available in planar form), the kernel mean values $\bar{K}(v)$ in planar approximation can be directly used as a replacement of the point values $K(x, y)$. From Table 2, the geodetic kernels considered are represented in the inner zone through three different asymptotic functions: inverse distance $(\sim 1 / \psi)$, inverse square distance $\left(\sim 1 / \psi^{2}\right)$ and inverse cubic distance $\left(\sim 1 / \psi^{3}\right.$ or $\left.\sim 1 / l^{3}\right)$; the higher the order of the asymptotic function, the larger the differences between mean and point kernels near the innermost zone. 
Table 2 Geodetic integral kemels, asymptotic and planar approximations and antiderivatives of planar approximation

\begin{tabular}{|c|c|c|c|c|c|}
\hline Integral name & $\begin{array}{c}\text { Kern } \\
\text { el }\end{array}$ & Factor c & $\begin{array}{c}\text { Asymptotic } \\
\text { approximation }\end{array}$ & $\begin{array}{c}\text { Planar } \\
\text { approximation }\end{array}$ & $\begin{array}{c}\text { Antiderivative of planar } \\
\text { approximation }\end{array}$ \\
\hline $\begin{array}{c}\text { Stokes-class } \\
\text { Stokes (The original) } \\
\text { Hotine } \\
\text { Eötvös } \\
\text { Green-Molodensky } \\
\text { Tidal displacement } \\
\text { Gravity OTL }\end{array}$ & $\begin{array}{l}S(\psi) \\
A(\psi) \\
B(\psi) \\
G(\psi) \\
L(\psi) \\
F^{\prime}(\psi)\end{array}$ & $\begin{array}{l}2 \\
2 \\
2 \\
6 \\
\sqrt{2} \\
-0.5\end{array}$ & $c / \psi$ & $\left.\begin{array}{c}K_{S} \\
K_{A} \\
K_{B} \\
K_{G} \\
K_{L} \\
K_{F}\end{array}\right\}=c\left(x^{2}+y^{2}\right)^{-1 / 2}$ & $\left.\begin{array}{l}F_{S} \\
F_{A} \\
F_{B} \\
F_{G} \\
F_{L} \\
F_{F}\end{array}\right\}=\begin{array}{c}c\left[x \ln \left(y+\sqrt{\left(x^{2}+y^{2}\right)}\right.\right. \\
+y \ln \left(x+\sqrt{\left.\left(x^{2}+y^{2}\right)\right]}\right.\end{array}$ \\
\hline Deflection-geoid & $C^{\prime}(\psi)$ & -2 & $c / \psi$ & $\left\{\begin{array}{l}K_{C^{\prime}}^{\xi} \\
K_{C^{\prime}}^{\prime \prime}\end{array}\right\}=c\left(x^{2}+y^{2}\right)^{-1}\left\{\begin{array}{l}y \\
x\end{array}\right\}$ & $\left\{\begin{array}{l}F_{C^{\prime}}^{\prime} \\
F_{C^{\prime}}^{\prime \prime}\end{array}\right\}=\left\{\begin{array}{l}c\left[x / 2 \ln \left(x^{2}+y^{2}\right)+\right. \\
y \arctan (x / y)-x] \\
c\left[y / 2 \ln \left(x^{2}+y^{2}\right)+\right. \\
x \arctan (y / x)-y]\end{array}\right\}$ \\
\hline $\begin{array}{l}\text { Vening-Meinesz-class } \\
\text { Vening-Meinesz } \\
\text { (The original) } \\
\text { Inverse } \\
\text { Vening-Meinesz }\end{array}$ & $\begin{array}{l}V(\psi) \\
H^{\prime}(\psi)\end{array}$ & -2 & $c / \psi^{2}$ & $\begin{array}{l}\left\{\begin{array}{l}K_{V}^{\xi}(x, y) \\
K_{V}^{\eta}(x, y)\end{array}\right\}=c\left(x^{2}+y^{2}\right)^{-3 / 2}\left\{\begin{array}{l}y \\
x\end{array}\right\} \\
\left\{\begin{array}{l}K_{H}^{\xi}(x, y) \\
K_{H}^{n}(x, y)\end{array}\right\}=c\left(x^{2}+y^{2}\right)^{-3 / 2}\left\{\begin{array}{l}y \\
x\end{array}\right\}\end{array}$ & $\begin{array}{l}\left\{\begin{array}{l}F_{V}^{\xi} \\
F_{V}^{\prime \prime}\end{array}\right\}=\left\{\begin{array}{l}-c \ln \left(x+\sqrt{x^{2}+y^{2}}\right) \\
-c \ln \left(y+\sqrt{x^{2}+y^{2}}\right)\end{array}\right\} \\
\left\{\begin{array}{l}F_{H^{\prime}}^{\xi} \\
F_{H^{\prime}}^{n}\end{array}\right\}=\left\{\begin{array}{l}-c \ln \left(x+\sqrt{x^{2}+y^{2}}\right) \\
-c \ln \left(y+\sqrt{x^{2}+y^{2}}\right)\end{array}\right\}\end{array}$ \\
\hline $\begin{array}{c}\text { Inverse-Stokes-Hotine-class } \\
\text { Inverse Stokes } \\
\text { Inverse Hotine }\end{array}$ & $E(\psi)$ & 8 & $c / \psi^{3}$ & $K_{E}=c\left(x^{2}+y^{2}\right)^{-3 / 2}$ & $F_{E}=-c \sqrt{x^{2}+y^{2}} /(x y)$ \\
\hline $\begin{array}{l}\text { Terrain-effect-class } \\
\text { Terrain correction } \\
\text { Indirect effect } \\
\text { Molodensky } G_{1}\end{array}$ & $T(l)$ & 1 & $c / l^{3}$ & $K_{T}=c\left(x^{2}+y^{2}\right)^{-3 / 2}$ & $F_{T}=-c \sqrt{x^{2}+y^{2}} /(x y)$ \\
\hline Poisson & $D(l)$ & 1 & $c / l^{3}$ & $K_{D}=c z_{0}\left(x^{2}+y^{2}+z_{0}^{2}\right)^{-3 / 2}$ & $F_{D}=c \arctan \left[(x y) /\left(z_{0} \sqrt{x^{2}+y^{2}+z_{0}^{2}}\right)\right]$ \\
\hline
\end{tabular}

\section{[Please insert Table2.doc here and delete this picture]}

a

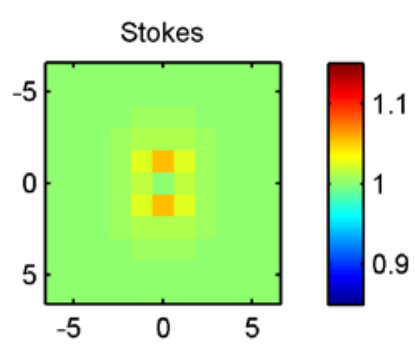

d

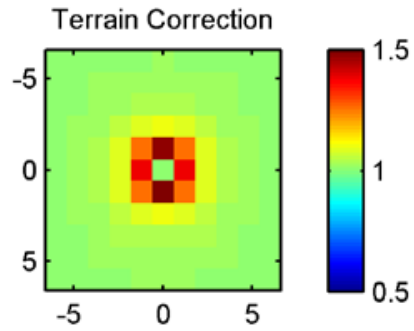

b

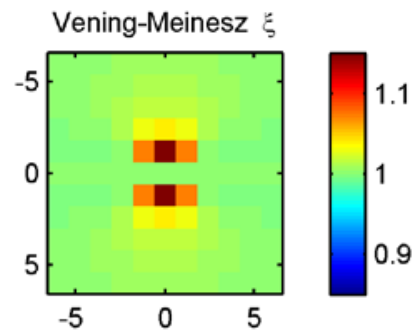

C Vening-Meinesz $\eta$

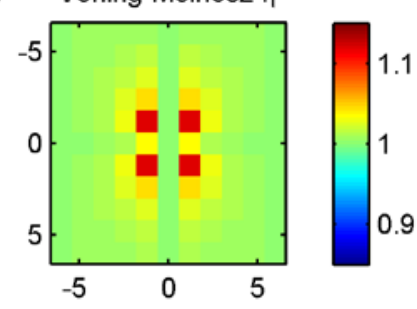

e

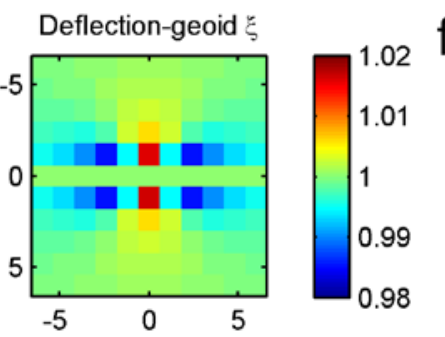

Deflection-geoid $\eta$

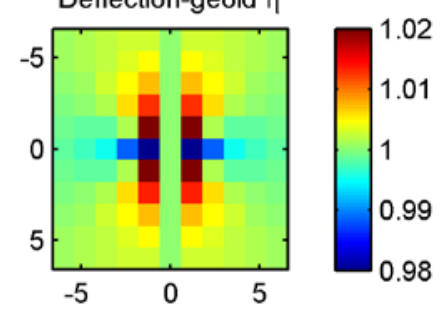

Fig 9 Kernel weighting factors $\bar{K}(v) / K(x, y)$ for a) Stokes, b) Vening-Meinesz $\xi$ component, c) Vening-Meinesz $\eta$-component, d) Terrain correction, e) deflection-geoid $\xi$ component, f) deflection geoid $\eta$-component. Latitude of central parallel is $-35^{\circ}$, grid 
resolution is 1 arc-min. Each pannel shows the innermost 11 x 11 cells centred on the computation point. The innermost zone contributions are set to zero.

To further illustrate this behaviour for some of the geodetic integrals compiled in Table 2, some kernel weighting factors $\bar{K}(v) / K(x, y)$ were computed and are shown in Fig. 9. For Stokes's function (Fig. 9a), the maximum kernel weighting factor is 1.06, showing that point and mean kernels differ by as much as 6\%. For Vening-Meinesz's kernel, the maximum kernel weighting factors are even larger (15\%). Relatively small maximum differences between mean and point kernels are found for the deflection-geoid kernel (about $2 \%$ ). The terrain effect kernel weighting factors are as large as $50 \%$ near the innermost zone (Fig. 9d), which is related to the high-order of the asymptotic representation (cf. Featherstone and Olliver 1997).

\section{Summary, conclusions and recommendations}

We investigated the computation of whole-of-cell mean values of integration kernels in geodetic convolution integrals and demonstrated their benefits with a detailed geodetic casestudy on the numerical evaluation of Stokes's integral. The analytical approach computes kernel weighting factors through a 2D analytical integration of the integral kernel over the surface area of the cell. The 2D analytical integration is performed with aid of planar approximations of the integral kernel. Kernel weighting factors are used to convert point to cell mean values of the non-planar kernel. The numerical approach is based on GaussLegendre quadrature (GLQ). This efficient numerical integration technique evaluates the kernel function at the zero-crossings of the Legendre polynomials and applies a special weighting scheme to yield accurate mean kernel estimates. It was shown that the computational requirements in GLQ are very low, i.e., 4-9 evaluations per cell are necessary to obtain sufficiently accurate mean kernels.

Mean kernels are a key issue in the accurate numerical evaluation of geodetic and other convolution integrals, as was demonstrated exemplarily by closed-loop tests for Stokes's integral (Sect 3.5). These tests were based on self-consistent sets of EGM2008 gravity anomalies and geoid heights. Compared to point kernel results, GLQ numerical and 2D analytical integration of mean kernels reduced the closed-loop RMS differences by a factor of $\sim 5$. The case study also assessed the performance of $1 \mathrm{D}$ and $2 \mathrm{D}$ analytical integration, showing the deficiencies of the $1 \mathrm{D}$ technique.

Any of the mean kernel approaches described here approximate 'true' mean kernels to some extent. With numerical mean kernels, the level of approximation is related to the finite number of evaluation points. With analytical mean kernels, the approximation originates from the planarisation of the kernel in the vicinity of the computation point. However, the equally good performance of the numerical and 2D analytical integration mean kernel gives 
strong evidence that these approximations are acceptable for accurate practical evaluation of convolution integrals.

As a general recommendation, mean kernels should be routinely preferred over point (centre-of-cell) kernels to accurately evaluate convolution integrals. The numerical and the 2D analytical integration approach are well-suited mean kernel computation methods. Both are easy to implement in geodetic convolution integral software and the additional computation time is very low. Such a use of mean kernels avoids one unnecessary approximation in the numerical evaluation of convolution integrals.

Acknowledgements: Christian Hirt is supported under the ARC's Discovery Projects funding scheme (project number DP0663020). Will Featherstone was the recipient of an Australian Research Council (ARC) Professorial Fellowship (project number DP0663020). The views expressed herein are those of the authors and are not necessarily those of the ARC. This work was also supported by iVEC (http://www.ivec.org/) through the use of advanced computing resources provided by the SGI Altix facility located at Technology Park, Perth, Australia. Some of our figures were produced using the Generic Mapping Tools (GMT; Wessel and Smith 1998). Thanks go to Tom Schramm (HCU Hamburg) for a discussion of analytical integration methods. We are grateful to the reviewers for their valuable comments and for directing us to the GLQ approaches. This is The Institute for Geoscience Research (TIGeR) publication number XXX. Postscriptum: During the review of this manuscript, reviewer 2 made the following remark "A curious question: Why did it take 20 years to come up with such a paper?" Our light-hearted response is "We were still only students back then!”

\section{Appendix A1: Gauss-Legendre quadrature}

Gauss-Legendre quadrature (GLQ; also abbreviated to Gaussian quadrature) is a numerical integration method that is suited to the approximate solution of definite integrals (e.g, Stark 1970). GLQ is one of the most efficient numerical integration methods, i.e., it needs a very small number of evaluation points for a rapid convergence of the numerical integration estimates (e.g., Sormann 2009). GLQ uses specially selected evaluation points $x_{i}$, at which the function $f(x)$ is evaluated, and applies weights $w_{i}$ to the function value $f\left(x_{i}\right)$ (Stoer and Bulirsch 1980).

Detailed descriptions of GLQ techniques are available in the mathematical literature (e.g., Stark 1970; Abramowitz and Stegun 1972; Conte and de Boor 1972; Engels 1980; Stoer and Bulirsch 1980; Hamming 1986). Here we summarise GLQ for numerical evaluation of $1 \mathrm{D}$ and 2D functions. The description is similar to the one given by Wild-Pfeiffer (2008). Finally, the 2D GLQ is adapted for the computation of geodetic mean kernels.

\section{A1: 1D Gauss-Legendre quadrature}


Within the unit interval [-1 1], the integral of a function $f(x)$ can be approximated by Gauss’s quadrature formula (Abramowitz and Stegun 1972, p 887)

$$
\int_{-1}^{1} f(x) d x \approx \sum_{i=1}^{n} w_{i}^{*} f\left(x_{i}\right)
$$

where $n$ is the quadrature degree, $w_{i}^{*}$ are weights and $x_{i}$ is the $i$ th zero-crossing of the $n$-th order Legendre polynomial $P_{n}(x)$. The weights are computed by (e.g., Abramowitz and Stegun 1972, p887)

$$
w_{i}^{*}=\frac{2}{\left(1-x_{i}^{2}\right)\left[P_{n}^{\prime}\left(x_{i}\right)\right]^{2}}
$$

where $P_{n}^{\prime}(x)$ is the first derivative of the $n$-th Legendre polynomial, which can be obtained from well-known recursive relations. The zero-crossings $x_{i}$ of the Legendre polynomial can be found by solving for the eigenvalues of the symmetric tridiagonal Jacobi matrix (e.g., Golub and Welsch 1969), or by application of Newton-Raphson iteration on asymptotic approximations (e.g., Lether and Wenston 1995). The zero-crossings $x_{i}$ and weights $w_{i}^{*}$ can be computed using publicly available software (e.g., van Winckel 2004). Table A1 lists the $w_{i}^{*}$ and $x_{i}$ values for quadratures with $n=1$ to 7 .

For the numerical evaluation within an arbitrary integration interval [a b], the integral of the function $f(x)$ is approximated by (Abramowitz and Stegun 1972, p 887):

$$
\int_{a}^{b} f(x) d x \approx \sum_{i=1}^{n} w_{i} f\left(s_{i}\right)
$$

where the transformed zero-crossings $s_{i}$ of the Legendre polynomials and weights $w_{i}$ are computed through linear mapping from unit interval [-1 1] to interval [a b] (after Abramowitz and Stegun 1972):

$$
\begin{aligned}
& s_{i}=\frac{b-a}{2} x_{i}+\frac{b+a}{2} \\
& w_{i}=\frac{b-a}{2} w_{i}^{*}
\end{aligned}
$$

Table A1 Zero crossings $x_{i}$ and weights $w_{i}^{*}$ for Gauss-Legendre quadratures to degree 7 (cf. Abramowitz and Stegun 1972, p 916; Stark 1970 p 224). Values rounded to 10 decimals.

\begin{tabular}{|c|r|c|}
\hline Degree of quadrature $n$ & zero crossings $x_{i}$ & weights $w_{i}^{*}$ \\
\hline 1 & $x_{1}=0.0000000000$ & $w_{1}^{*}=2.0000000000$ \\
\hline
\end{tabular}




\begin{tabular}{|c|c|c|}
\hline 2 & $-x_{2}=x_{1}=0.5773502692$ & $w_{1}^{*}=w_{2}^{*}=1.0000000000$ \\
\hline 3 & $\begin{array}{r}-x_{3}=x_{1}=0.7745966692 \\
x_{2}=0.0000000000\end{array}$ & $\begin{aligned} w_{1}^{*}=w_{3}^{*} & =0.5555555556 \\
w_{2}^{*} & =0.8888888889\end{aligned}$ \\
\hline 4 & $\begin{array}{l}-x_{4}=x_{1}=0.8611363116 \\
-x_{3}=x_{2}=0.3399810436\end{array}$ & $\begin{array}{l}w_{1}^{*}=w_{4}^{*}=0.3478548451 \\
w_{2}^{*}=w_{3}^{*}=0.6521451549\end{array}$ \\
\hline 5 & $\begin{aligned}-x_{5}=x_{1} & =0.9061798459 \\
-x_{4}=x_{2} & =0.5384693101 \\
x_{3} & =0.0000000000\end{aligned}$ & $\begin{aligned} w_{1}^{*}=w_{5}^{*} & =0.2369268851 \\
w_{2}^{*}=w_{4}^{*} & =0.4786286705 \\
w_{3}^{*} & =0.5688888889\end{aligned}$ \\
\hline 6 & $\begin{array}{l}-x_{6}=x_{1}=0.9324695142 \\
-x_{5}=x_{2}=0.6612093865 \\
-x_{4}=x_{3}=0.2386191861\end{array}$ & $\begin{array}{l}w_{1}^{*}=w_{6}^{*}=0.1713244924 \\
w_{2}^{*}=w_{5}^{*}=0.3607615730 \\
w_{3}^{*}=w_{4}^{*}=0.4679139346\end{array}$ \\
\hline 7 & $\begin{array}{r}-x_{7}=x_{1}=0.9491079123 \\
-x_{6}=x_{2}=0.7415311856 \\
-x_{5}=x_{3}=0.4058451514 \\
x_{4}=0.0000000000\end{array}$ & $\begin{array}{r}w_{1}^{*}=w_{7}^{*}=0.1294849662 \\
w_{2}^{*}=w_{6}^{*}=0.2797053915 \\
w_{3}^{*}=w_{5}^{*}=0.3818300505 \\
w_{4}^{*}=0.4179591837\end{array}$ \\
\hline
\end{tabular}

\section{A2: 2D Gauss-Legendre Quadrature}

When applied to 2D numerical integration, GLQ is sometimes also named cubature (e.g., Stroud 1971, Engels 1980). Using the unit square [-1 1] $\times[-11]$ as the integration domain, the double integral of the function $f(x, y)$ is approximated as (Stroud 1971, Wild-Pfeiffer 2008):

$$
\int_{-1}^{1} \int_{-1}^{1} f(x, y) d x d y \approx \sum_{i=1}^{n} \sum_{j=1}^{m} w_{i}^{*} w_{j}^{*} f\left(x_{i}, y_{j}\right)
$$

where $n$ is the degree of the cubature in $x, m$ is the degree of the cubature in $y$ (for simplicity, we use $n=m), x_{i}$ is the $i$-th zero-crossing of the $n$-th order Legendre polynomial $P_{n}(x), y_{i}$ is the $j$-th zero-crossing of the $n$-th Legendre polynomial $P_{n}(y)$ and $w_{i}^{*}, w_{j}^{*}$ are the weights 
(Eq. A2 and Table A1). In analogy to the 1D case, the integral of the function $f(x, y)$ can be computed for arbitrary integration domains $[\mathrm{a} b] \times[\mathrm{c} d]$ using

$$
\int_{a}^{b} \int_{c}^{d} f(x, y) d x d y \approx \sum_{i=1}^{n} \sum_{j=1}^{m} w_{i} w_{j} f\left(s_{i}, t_{j}\right)
$$

where $s_{i}, t_{j}$ are the (transformed) zero-crossings and weights $w_{i}, w_{j}$. The quantities $t_{i}$ and $w_{j}$ are obtained through [cf. Eqs (A4-A5)]:

$$
\begin{gathered}
t_{j}=\frac{d-c}{2} y_{j}+\frac{d+c}{2} \\
w_{j}=\frac{d-c}{2} w_{j}^{*}
\end{gathered}
$$

Finally, the 2D GLQ is slightly modified so that it can be used in the numerical computation of cell-mean values of Stokes's function and other geodetic kernels. As integration domain $[\mathrm{a} \mathrm{b}] \times[\mathrm{c} d]$, we use $\left[\lambda_{1} \lambda_{2}\right] \times\left[\varphi_{1} \varphi_{2}\right]$, bounding the cell under evaluation. The 2D function $f(x, y)$ is the geodetic kernel function $Y(\psi)$, which depends on the spherical distance $\psi$ [being a $2 \mathrm{D}$-function of the computation point $\left(\varphi_{P}, \lambda_{P}\right)$ and evaluation point $\left(\varphi_{Q^{\prime} i,} \lambda_{Q^{\prime} j}\right)$, cf. Eq. 2]. The 2D GLQ for numerical computation of whole-of-cell mean kernels then reads:

$$
\int_{\lambda_{1}}^{\lambda_{\varphi_{1}}} \int_{\varphi_{2}} Y(\psi) \cos (\varphi) d \varphi d \lambda \approx \frac{1}{\sum w_{i} w_{j}} \sum_{i=1}^{n} \sum_{j=1}^{m} w_{i} w_{j} Y\left(\psi\left(\varphi_{P,} \lambda_{P}, \varphi_{Q^{\prime} j}, \lambda_{Q^{\prime} i}\right)\right)
$$

where the evaluation points $\left(\varphi_{Q^{\prime} i}, \lambda_{Q^{\prime} j}\right)$ located inside the integration domain $\left[\lambda_{1} \lambda_{2}\right] \times\left[\varphi_{1} \varphi_{2}\right]$. The coordinates $\left(\varphi_{Q^{\prime} i}, \lambda_{Q^{\prime} j}\right)$ are computed as a function of the cell boundaries $\left(\lambda_{1}, \lambda_{2}, \varphi_{1}, \varphi_{2}\right)$ and as a function the zero-crossings $x_{i}, y_{j}$ of the $n$-th order Legendre polynomial through:

$$
\begin{aligned}
& \lambda_{Q^{\prime} i}=\frac{\lambda_{2}-\lambda_{1}}{2} x_{i}+\frac{\lambda_{2}+\lambda_{1}}{2} \\
& \varphi_{Q^{\prime} j}=\frac{\varphi_{2}-\varphi_{1}}{2} y_{j}+\frac{\varphi_{2}+\varphi_{1}}{2}
\end{aligned}
$$

The weights $w_{i} w_{j}$ are computed as a function of the cell boundaries $\left(\lambda_{1}, \lambda_{2}, \varphi_{1}, \varphi_{2}\right)$ and as a function of $w_{i}^{*}, w_{j}^{*}$ (Eq. A2):

$$
\begin{aligned}
& w_{i}=\frac{\lambda_{2}-\lambda_{1}}{2} w_{i}^{*} \\
& w_{j}=\frac{\varphi_{2}-\varphi_{1}}{2} w_{j}^{*}
\end{aligned}
$$


It should be noted that the numerical integration result from Eq. (A10) is divided by the sum of the weights $w_{i} w_{j}$ applied, so that the cell-mean value of Stokes function is obtained (and not a volume). The required GLQ parameters $x_{i}, w_{i}^{*}$ (and $y_{j}, w_{j}^{*}$, respectively) can be computed with existing software. Examples are the routine gauleg (Numerical Recipes in C, Press et al. 2002, p 152; Numerical Recipes in Fortran, Press et al. 2003, p 145), and the Matlab function lgwt (von Winckel 2004).

\section{References}

Abramowitz MA Stegun IA (1972) Handbook of mathematical functions. Dover Publications, New York.

Alberts B, Klees R (2004) A comparison of methods for the inversion of airborne gravity data. J Geod 78(1): 55-65. DOI 10.1007/s00190-003-0366-X

Asgharzadeh MF, von Frese RB, Kim HR, Leftwich TE, Kim JW (2007) Spherical prism gravity effects by Gauss-Legendre quadrature integration. Geophysical Journal International 169(1): 1-11. DOI: 10.1111/j.1365-246X.2007.03214.x

Bláha T, Hirsch M, Keller W, Scheinert M (1996) Application of a spherical FFT approach in airborne gravimetry, J Geod 70(11): 663-672, doi: 10.1007/BF00867145

Bos MS, Baker TF (2005) An estimate of the errors in gravity ocean tide loading computations, J Geod 79(1-3): 50-63, doi: 10.1007/s00190-005-0442-5

Boyarsky EA, Afanasyeva LV, Koneshov, VN, Rozhkov YE (2010) On the calculation of the vertical deflection and the geoid undulation from gravity anomalies. Physics of the Solid Earth 46(6): 538-543.

Claessens SJ, Hirt C, Amos MJ, Featherstone WE, Kirby JF (2011) The NZGEOID09 New Zealand quasigeoid model, Survey Review 43(319): 2-15, doi: 10.1179/003962610X12747001420780

Conte SD, de Boor C (1972) Elementary numerical analysis - an algorithmic approach. McGraw-Hill, Kogakusha.

de Min E (1994) On the numerical evaluation of Stokes’ integral, International Geoid Service Bulletin 3: 41-46

de Min E (1996) De Geoide voor Nederland. Nederlandse Commissie voor Geodesie Publikatie 34 (Dutch Geodetic Commission Publication No 34), Delft.

El Habiby MM (2007) Wavelet Representation of Geodetic Operators, UCGE Report No 20250, University of Calgary.

Engels H (1980), Numerical Quadrature and Cubature, London, Academic Press

Featherstone WE (2002) Tests of two forms of Stokes's integral using a synthetic gravity field based on spherical harmonics, in: Grafarend EW, Krumm FW, Schwarze VS (eds.) Geodesy - The Challenge for the Third Millennium, Springer, Berlin Heidelberg New York, pp 163-171

Featherstone WE, Kirby JF, Hirt C, Filmer MS, Claessens SJ, Brown N, Hu, G, Johnston GM (2010) The AUSGeoid2009 model of the Australian Height Datum, J Geod Online First. doi 10.1007/s00190-010-0422-2

Featherstone WE, Olliver JG (1997) A method to validate gravimetric geoid computation software based on Stokes's integral, J Geod 71(9): 571-576, doi:

$10.1007 / \mathrm{s} 001900050125$ 
Forsberg R (1984) A study of terrain reductions, density anomalies and geophysical inversion methods in gravity field modelling, Report 355, Department of Geodetic Science and Surveying, Ohio State University, Columbus

Forsberg R (1985) Gravity field terrain effect computations by FFT, Bulletin Géodésique 59(4): 342-360, doi: 10.1007/BF02521068

Golub GH, Welsch JH (1969) Calculation of Gauss quadrature rules. Math Comp 23(106): 221-230+s1-s10

Haagmans R, de Min E, van Gelderen M (1993) Fast evaluation of convolution integrals on the sphere using 1D FFT, and a comparison with existing methods for Stokes' integral, manuscripta geodaetica 18(5): 227-241

Hammer S (1939) Terrain corrections for gravimeter stations, Geophysics 4(3): 184-194, doi:10.1190/1.1440495

Hamming RW (1986) Numerical methods for scientists and engineers, second edition. Reprint, Dover publications.

Heiskanen, WA, Moritz H (1967) Physical Geodesy, Freeman, San Francisco

Hipkin RG (1988) Bouguer anomalies and the geoid: a reassessment of Stokes's method, Geophysical Journal International 92: 53-66, doi: 10.1111/j.1365-246X.1988.tb01120.x

Holmes, SA, Pavlis NK (2008) Spherical harmonic synthesis software harmonic_synth. http://earth-info.nga.mil/GandG/wgs84/gravitymod/new_egm/new_egm.html

Hotine M (1969) Mathematical Geodesy, ESSA Monograph No 2, US Department of Commerce, Washington DC

Huang J, Vaníček P, Novák P (2000) An alternative algorithm to FFT for the numerical evaluation of Stokes’s integral, Studia Geophysica et Geodaetica 44(3): 374-380, doi: 10.1023/A:1022160504156

Hwang C (1998) Inverse Vening Meinesz formula and deflection-geoid formula: applications to the predictions of gravity and geoid over the South China Sea, J Geod 72(5): 304312, doi: 10.1007/s001900050169

Kearsley AHW (1986) Data Requirements for Determining Precise Relative Geoid Heights From Gravimetry. Journal of Geophysical Research 91(B9): 9193-9201

Klees R (1996) Numerical calculation of weakly singular surface integrals. J Geod 70(11): 781-797

Lehmann R (1997) Fast space-domain evaluation of geodetic surface integrals J Geod 71(9): 533-540

Lether FG, Wenston PR (1995) Minimax approximation to the zeros of P_n(x) and GaussLegendre quadrature. J Comp Appl Math 59(2): 245-252, doi:10.1016/03770427(94)00030-5

Makhloof AA, Ilk KH (2008) Far-zone effects for different topographic-compensation models based on a spherical harmonic expansion of the topography. J Geod 82(10): 613-635

Molodensky MS, Yeremeyev VF, Yurkina MI (1962) Methods for study of the external gravitational field and figure of the Earth. Translated from Russian, Isreali Programme for Scientific Translations, Jerusalem

Moritz H (1980) Advanced physical geodesy. Wichmann Verlag, Karlsruhe

Newton’s Bulletin (2009), Newton’s Bulletin Issue n 4, April 2009 ISSN 1810-8555, Publication of the International Association of Geodesy and International Gravity Field Service 
Novák P, Vaníček P, Véronneau M, Holmes SA, Featherstone WE (2001) On the accuracy of modified Stokes's integration in high-frequency gravimetric geoid determination, $\mathrm{J}$ Geod 74(9): 644-654, doi: 10.1007/s001900000126

Pavlis NK, Holmes SA, Kenyon SC, Factor JK (2008) An Earth Gravitational Model to Degree 2160: EGM2008, Presented at the 2008 General Assembly of the European Geoscience Union, Vienna, Austria, April 13-18, 2008

Press WH, Teukolsky SA, Vetterling WT, Flannary BP (2003) Numerical Recipes in Fortran 77 - The Art of Scientific Computing, Second Edition. Volume 1 of Fortran Numerical Recipes. Cambridge University Press, Cambridge

Press WH, Teukolsky SA, Vetterling WT, Flannary BP (2002) Numerical Recipes in Fortran C - The Art of Scientific Computing, Second Edition. Cambridge University Press, Cambridge

Sampietro D, Sona G, Venuti G (2007) Residual Terrain Correction on the Sphere by an FFT Algorithm. In: Proceed. 1st Intern. Sympos. International Gravity Field Service, Aug 28 - Sep 1, Istanbul, Turkey, Harita Dergesi Special Issue, pp 306-311

Schwarz K-P, Sideris MG, Forsberg R (1990) The use of FFT techniques in physical geodesy, Geophys J Int 100(3): 485-514, doi: 10.1111/j.1365-246X.1990.tb00701.x Sideris MG, Li YC (1993) Gravity field convolution without windowing and edge effects, Bulletin Géodésique 67(2):107-118, doi: 10.1007/BF01371374

Sideris MG, She BB (1995) A new high-resolution geoid for Canada and part of the US by the 1D-FFT method, Bulletin Géodésique 69(2): 92-108, doi: 10.1007/BF00819555

Sormann H (2009) Numerische Methoden in der Physik Institut für Theoretische Physik Computational Physics, TU Graz, Austria. URL: http://itp.tugraz.at/LV/sormann /NumPhysik/Skriptum/

Stark PA (1970) Introduction to numerical methods. The MacMillian Company, New York

Stoer J, Bulirsch R (1980) Introduction to numerical analysis. Springer, New York

Strang van Hees G (1990) Stokes formula using Fast Fourier Techniques, manuscripta geodaetica 15(4): 235-239.

Stroud AH (1971) Approximate Calculation of Multiple Integrals. Prentice-Hall, New York

Torge W (2001) Geodesy, third edition, De Gruyter, Berlin New York

Tscherning CC (2003) Proposal for the precise definition of mean values of gravity field quantities. In: Newton's Bulletin, No. 1, pp. 11-13, International Geoid Service

Tziavos IN (1996) Comparisons of spectral techniques for geoid computations over large areas, J Geod 70(6): 357-373, doi: 10.1007/BF00868188

Val'ko M, Mojzeš M, Janák J, Papčo J (2008) Comparison of two different solutions to Molodensky’s $\mathrm{G}_{1}$ term, Studia Geophysica et Geodaetica 52(1): 71-86. doi: 10.1007/s11200-008-0006-2

van Gelderen (1991) The geodetic boundary value problem in two dimensions and its iterative solution, Publication No 35, Netherlands Geodetic Commission, Amsterdam

van Gysen H (1994) Thin-plate spline quadrature of geodetic integrals. Bulletin Geodesique 68: $173-179$

Vaníček P, Kleusberg A (1987) The Canadian geoid - Stokesian approach, manuscripta geodaetica 12(2):86-98

Vaníček P, Krakiwsky EJ (1986) Geodesy: The concepts, Elsevier, Amsterdam New York

Vening-Meinesz FA (1928) A formula expressing the deflection of the plumb-lines in the gravity anomalies and some formulae for the gravity field and the gravity potential outside the geoid. Proc. Koninkl. Akad. Wetenschaft 31: 315-331, Amsterdam 
von Winckel G (2004) Legendre-Gauss Quadrature Weights and Nodes. Matlab function lgwt . URL: http://www.mathworks.com/matlabcentral/fileexchange/4540Wessel P, Smith WHF (1998), New, improved version of the Generic Mapping Tools released, EOS Trans. AGU, 79: 579.

Wild-Pfeiffer F (2008) A comparison of different mass elements for use in gravity gradiometry. J Geod 82(10): 637-653. doi: 10.1007/s00190-008-0219-8

Zhang C (1993) Recovery of gravity information from satellite altimetry data and associated forward geopotential models, UCGE Report No 20058, University of Calgary

Zhang C (1995) A general formula and its inverse formula for gravimetric transformations by use of convolution and deconvolution techniques, J Geod 70(1-2): 51-64, doi: 10.1007/BF00863418 